Published in final edited form as:

Nat Struct Mol Biol. 2019 October ; 26(10): 946-954. doi:10.1038/s41594-019-0304-0.

\title{
Cryo-EM structure of the CIpXP protein degradation machinery
}

\author{
C Gatsogiannis ${ }^{\# 1}$, D Balogh ${ }^{\# 2}$, F Merino1, SA Sieber ${ }^{2, b}$, S Raunser ${ }^{1, b}$ \\ ${ }^{1}$ Department of Structural Biochemistry, Max Planck Institute of Molecular Physiology, Dortmund, \\ Germany \\ ${ }^{2}$ Department of Chemistry, Chair of Organic Chemistry II, Center for Integrated Protein Science \\ (CIPSM), Technische Universität München, Garching, Germany \\ \# These authors contributed equally to this work.
}

\begin{abstract}
The ClpXP machinery is a two component protease complex performing targeted protein degradation in bacteria and mitochondria. The complex consists of the AAA+ chaperone $\mathrm{ClpX}$ and the peptidase ClpP. The hexameric ClpX utilizes the energy of ATP binding and hydrolysis to engage, unfold and translocate substrates into the catalytic chamber of tetradecameric $\mathrm{ClpP}$ where they are degraded. Formation of the complex involves a symmetry mismatch, since hexameric $\mathrm{AAA}+$ rings bind axially to the opposing stacked heptameric rings of the tetradecameric ClpP. Here we present the cryo-EM structure of ClpXP from Listeria monocytogenes. We unravel the heptamer-hexamer binding interface and provide novel insights into the $\mathrm{ClpX}-\mathrm{ClpP}$ crosstalk and activation mechanism. The comparison with available crystal structures of $\mathrm{ClpP}$ and $\mathrm{ClpX}$ in different states allows us to understand important aspects of ClpXP's complex mode of action and provides a structural framework for future pharmacological applications.
\end{abstract}

\section{Introduction}

Caseinolytic protease $\mathrm{P}(\mathrm{ClpP})$ represents a major proteolytic protein in prokaryotes and in organelles of eukaryotes which is involved in protein homeostasis, bacterial pathogenesis as well as cancer progression ${ }^{1-3}$. ClpP is highly conserved, essential for virulence and regulation of stress responses in several pathogenic bacteria and therefore considered as a

\footnotetext{
Users may view, print, copy, and download text and data-mine the content in such documents, for the purposes of academic research, subject always to the full Conditions of use:http://www.nature.com/authors/editorial_policies/license.html\#terms

${ }^{\text {b }}$ To whom correspondence may be addressed. stephan.sieber@tum.de or stefan.raunser@mpi-dortmund.mpg.de.

Author Contributions S.A.S. and S.R. designed the study. C.G. screened and optimized samples, prepared cryo-EM grids, processed, and analyzed cryo-EM data. D.B. cloned, overexpressed and purified proteins, optimized sample preparation, conducted activity assays and gel filtration measurements, analyzed HDX-MS data. C.G. and F.M. built atomic models. C.G. and D.B. prepared figures, C.G., D.B., S.A.S., S.R. wrote the manuscript. All authors discussed the results.

Competing Interests The authors declare no competing interests.

Reporting summary:

Further information on experimental design is available in the Nature Research Reporting Summary linked to this article.

Data availability:

The cryoEM map of LmClpXP1/2 has been deposited at the EMDB with the accession code EMD-10162. The corresponding molecular models of LmClpX and LmClpP1/2 have been deposited at the wwPDB with accession codes PDB 6SFW and PDB 6SFX, respectively. Source data for Figure 4, Figure 5b-c, Supplementary Figure 1a and Supplementary Figure 6 are available online. All data used in this study are available from the corresponding authors upon reasonable request.
} 
promising therapeutic target for novel antibiotics ${ }^{4}$. ClpP associates with diverse ATPdependent $\mathrm{AAA}+$ chaperones such as $\mathrm{ClpX}, \mathrm{ClpC}$ and $\mathrm{ClpA}$ to form a complex for the recognition, unfolding and digestion of substrate proteins ${ }^{5}$. To date, a large fraction of research has been dedicated to functionally exploit $\mathrm{ClpP}$ and its cognate chaperones, foremost ClpX, in terms of their enzymatic activity, individual structures and conformational control.

Previous low resolution electron microscopy (EM) studies of ClpXP and ClpAP from Escherichia coli revealed that up to two hexameric ClpX chaperones bind to a ClpP tetradecameric barrel ${ }^{6,7}$. The barrel consists of two stacked heptameric rings, forming a degradation chamber with 14 proteolytic sites ${ }^{8}$. Each ClpX subunit consists of an $\mathrm{N}$-terminal zinc binding domain (ZBD) and a C-terminal AAA+ domain. The ZBDs at the periphery of $\mathrm{ClpX}$ are responsible for recognition and engagement of several substrates ${ }^{9}$. ClpX hydrolyzes ATP to unfold the target substrates and translocate the unfolded polypeptides through a central pore into the proteolytic chamber of the ClpP barrel (for review see ${ }^{10}$ ).

Early on, the hexamer-heptamer ClpX-ClpP interface fascinated researchers and several studies characterizing the role of putative interaction motifs have led to models explaining the symmetry mismatch and functional interaction between the two proteins ${ }^{7,11-13}$. Sequence alignments and mutational studies of AAA+ chaperones identified loops in ClpX, that interact with the hydrophobic clefts on the periphery of ClpP. They contain the highly conserved (I/L/V)-G-(F/L) motif and are essential for complex formation ${ }^{14}$.

More recently, cyclic acyldespipeptides (ADEPs), a novel class of anti-bacterial compounds, have been identified to bind to the same peripheral hydrophobic clefts on ClpP and to induce the opening of the axial pores of $\mathrm{ClpP}^{4,15-17}$. They stabilize ClpP in an "open" activated state in the absence of the chaperone, leading to unregulated proteolysis of substrates and finally to cell death ${ }^{18}$. This suggests that the protruding loops in ClpX that contain the (I/L/V)-G-(F/L) motif, also called IGF loops, are sufficient to activate ClpP. It has also been speculated that this activation involves the opening of the axial pore to allow translocation of the substrate into the proteolytic chamber of ClpP. However, due to the lack of highresolution structures, a detailed understanding of the interaction between $\mathrm{ClpX}$ and $\mathrm{ClpP}$ is missing.

Contacts between the pore- 2 loops of $\mathrm{ClpX}$ and the $\mathrm{N}$-termini of ClpP represent a second set of well-characterized interactions between $\mathrm{ClpX}$ and $\mathrm{ClpP}$, which are, however, more dynamic and dependent on the nucleotide state of $\mathrm{ClpX}^{13}$. A crucial function of the $\mathrm{ClpP} \mathrm{N}$ termini is to gate the entrance of the proteolytic chamber ${ }^{11}$. Despite these detailed biochemical insights, a high-resolution structure of the whole proteolytic complex is lacking, thereby limiting our understanding of this important protein degradation machinery. Here we present a $4 \AA$ cryo-EM structure of ClpXP1/2 from Listeria monocytogenes. 


\section{Results}

\section{Cryo-EM structure of CIpXP1/2}

In order to obtain a ClpXP complex that is suitable for structural studies, we used the ClpP1/2 from $L$. monocytogenes. In contrast to other bacteria, $L$. monocytogenes encodes two ClpP isoforms, $\mathrm{LmClpP} 1$ and $\mathrm{LmClpP} 2$, which can assemble into heterooligomers composed of two homoheptameric rings. Recent studies have revealed that $\mathrm{ClpP} 1 / 2$ has a higher affinity to ClpX in comparison to the more conserved ClpP2 homocomplex ${ }^{19,20}$, suggesting a superior stability of the heterooligomer. As ClpP1/2 might cleave ClpX to a small extent during sample preparation, we mutated one residue of the catalytic triad (S98A) in both ClpP isoforms. Furthermore, we mutated the nucleotide binding site of ClpX (E183Q) to allow ATP binding, but to prevent hydrolysis, which results in a tighter binding to $\mathrm{ClpP}^{21,22}$.

We formed a complex of ClpX and ClpP1/2 and obtained a large fraction of ClpXP1/2 dimers (ClpP1/2-ClpX-ClpX-ClpP1/2) that were in equilibrium with ClpXP1/2 monomers (Supplementary Fig. 1a-c). It has been demonstrated before that two ClpX or ClpA hexamers can bind to one ClpP barrel from both sites, resulting in a ClpX-ClpP-ClpX or ClpA-ClpP-ClpA complex ${ }^{6,7,23}$. However, ClpXP1/2 dimers (Supplementary Figure 1a-d) have, to our knowledge, not been described so far. We therefore concentrated our structural analysis first on these intriguing dimers and determined their structure by cryo-EM and single particle analysis using crYOLO ${ }^{24}$ and SPHIRE $^{25}$ (Figure 1a-b, Supplementary Fig. 1e-g, Table 1). Although the intrinsic flexibility of the complexes did not allow the determination of a high-resolution structure (Supplementary Video 1, Supplementary Figure $1 \mathrm{e}-\mathrm{g}$ ), the fitting of the crystal structure of ClpX into the cryo-EM density suggests that the flexible $\mathrm{N}$-terminal zinc binding domains (ZBDs) of ClpX mediate the interaction between two ClpX hexamers (Figure 1c). While ZBD-deleted ClpX still associated with ClpP to a small extent, ClpX dimerization was completely abolished supporting our structural data (Supplementary Fig. 1a).

The ZBDs are involved in substrate binding and cofactor recognition and were shown to dimerize when expressed as single domain ${ }^{26,27}$. Based on these results it has been previously proposed that the ZBDs of neighboring subunits within a single ClpX hexamer dimerize resulting in a trimer-of-dimer model ${ }^{26}$. In this model the ZBD dimers interact with the adjacent dimers, creating a ring structure that is aligned with the central channel of ClpX. The structure of the ClpXP1/2 dimer, however, reveals that the ZBDs do not form rings, but arrange in a flexible half-cone spiral with the first and last ZBD dimer positioned directly above or at the rim of the axial pore entry of the upper and lower ClpX hexamer, respectively (Figure 1c, Supplementary Figure 1e). The ZBDs are apparently interacting with the ZBDs from oppositely positioned subunits leading to the cross-linking of the two opposing ClpX hexamers (Figure 1c, d). In total, four ZBD dimers fit into the cryo-EM density (Figure 1c). Because of the limited resolution in this region, however, we cannot determine if the cross-bridges are mediated by single ZBDs that dimerize with ZBDs of the other ClpX or by ZBD dimers that interact with dimers of the other ClpX. Based on these results and the fact that the ZBDs are flexible and not resolved in the crystal structure of 
$\mathrm{ClpX}^{28}$, we propose that ZBD dimers form stable structures only at the interface between two oppositely positioned ClpX hexamers (Figure 1d).

To obtain a cryo-EM structure at higher resolution, we focused the structural analysis on one ClpXP1/2 subunit in the dimer and solved its structure using the same dataset (Figure 2a-d, Supplementary Fig. 2, Table 1). The final cryo-EM reconstruction has an average resolution of 3.6 - $4 \AA$ for ClpP1/2 and 6 - $7 \AA$ for ClpX (Supplementary Fig. 2e-g). The overall lower resolution of $\mathrm{ClpX}$ indicates that the chaperone is intrinsically more flexible and heterogeneous than the ClpP barrel in the ClpXP1/2 complex. To build a complete atomic model of ClpXP1/2, we fitted a homology model of ClpX and the available crystal structure of ClpP1/2 (PDB-ID 4RYF) into the cryo-EM density and refined the model using Molecular Dynamics Flexible Fitting (MDFF) ${ }^{29}$.

The structure of ClpXP1/2 reveals that $\mathrm{ClpP} 1$ forms the upper homoheptamer of the $\mathrm{ClpP}$ barrel, whereas ClpP2 sits below and interacts with ClpX (Figure 2c-h). Our cryo-EM structure is consistent with previous binding studies on Listeria monocytogenes and Mycobacterium tuberculosis $\mathrm{ClpP}$ proteases, showing ClpX-ClpP1/2 interactions exclusively via the $\mathrm{ClpP} 2$ ring surface ${ }^{30-32}$.

Interestingly, the $\mathrm{ClpX}$ hexamer is not centrally aligned, but slightly tilted by $\sim 11^{\circ}$ towards $\mathrm{ClpP} 2$. The structure of $\mathrm{ClpP} 1 / 2$ is almost identical to the available crystal structure of apoClpP1/2 (PDB-ID 4RYF), indicating that the binding of ClpX does not induce large conformational changes in $\mathrm{ClpP} 1 / 2$. In contrast, interaction with $\mathrm{ClpP} 1 / 2$ has an effect on the overall conformation of ClpX. Whereas the crystal structure of E. coli ClpX shows the ATPase domains in a "dimer-of-trimers" arrangement ${ }^{33}$, our structure shows that upon ClpP1/2 binding, these domains become more regularly arranged and are related by pseudosix-fold symmetry. Unlike recent substrate bound AAA+ structures that show a "spiralstaircase" arrangement with one "seam" subunit moderately displaced from the pore ${ }^{34-36}$, all neighboring AAA+ domains of ClpX pack closely with each other. The resolution at the nucleotide pocket is not high enough to visualize nucleotides, but the structure reveals that all six ClpX protomers are in the "loadable" conformation (Supplementary Fig. 3). This is in contrast to ClpX with the E183Q mutation in its apo-state ${ }^{28,33}$. There, two subunits are in the "loadable" (L) and four are in the "unloadable" (U) conformation (Supplementary Fig. 3). In the L state, the arrangement of the small and large AAA+ domains results in an open binding cleft, to which the nucleotide can bind. In the U state, this site is blocked. A dynamic interconversion between $\mathrm{L}$ and $\mathrm{U}$ conformations is required to couple ATP hydrolysis by ClpX to mechanical work.

However, the arrangement is not a direct consequence of the bound nucleotide or the presence of specific mutations ${ }^{28}$. To further examine the interaction between ClpP1/2 and ClpX we used hydrogen-deuterium exchange with mass spectrometry (HDX-MS) to monitor the accessibility of residues at the interface. In line with our structural observations, complex formation between ClpP1/2 and ClpX only changes the accessibility of residues of ClpX and ClpP2, but not of ClpP1 (Supplementary Fig. 4). This not only corroborates that ClpX solely interacts with the $\mathrm{ClpP} 2$ isoform, but also indicates that $\mathrm{ClpX}$ binding does not induce major allosteric conformational changes in the $\mathrm{ClpP} 1$ heptamer. 


\section{Symmetry mismatch of IGF-loop interaction}

The most interesting part of the structure is the interface between ClpP2 and ClpX, which involves a C6/C7 symmetry mismatch. As predicted by biochemical studies ${ }^{8,12,14}$, it is mediated mainly by the flexible IGF loops of ClpX interacting with hydrophobic grooves in ClpP2 (Figure 2c-d, Supplementary Figure 5a). The tilted arrangement of ClpX results in part of the loops interacting stronger with ClpP2 than others (Figure 3a).

The large domains of the respective $\mathrm{ClpX}$ subunit from which the loops protrude are positioned directly below the deep hydrophobic grooves of $\mathrm{ClpP} 2$ which are formed at the interface of two subunits. This arrangement allows a direct interaction of the IGF-loops with the opposing grooves. The hydrophobic grooves of $\mathrm{ClpP}$ are arranged in a circular manner with seven-fold symmetry and the positions of the ClpX IGF-loops in the complex, perfectly match this arrangement. Interestingly, both rings display similar diameters (Figure 3b-c), except that the IGF-ring remains open at the position of the seventh, free hydrophobic cleft.

Five of the six IGF loops (subunits $\mathrm{O}, \mathrm{P}, \mathrm{R}, \mathrm{S}, \mathrm{T}$ ) display an overall similar arrangement. Due to the symmetry mismatch the large domain of the sixth subunit (subunit $Q$ ), is positioned in-between two hydrophobic grooves. The respective IGF-loop, however, still interacts with one of the opposing grooves by adopting an "extended" conformation (Figure $2 \mathrm{c}-\mathrm{h})$. The other groove stays empty. Although the distance between the IGF-loop and the "left" or "right" ClpP hydrophobic groove are similar, we only obtained a high-resolution structure with the IGF-loop binding exclusively to the left binding pocket.

To support our structural findings, we performed HDX-MS measurements and mutational studies. Upon complex formation deuterium uptake of the IGF-loop is strongly reduced (Figure 4, Supplementary Fig. 4) and mutations in the IGF loops of ClpX and the hydrophobic grooves of $\mathrm{ClpP} 2$ result in impaired complex formation (Supplementary Fig. 6). This is in line with our ClpXP1/2 structure that demonstrates that the interaction between the IGF loops with the hydrophobic grooves is crucial for complex formation and function.

Taken together, tilting of the ClpX ring and stretching of one of the IGF-loops is sufficient for the hexameric ClpX to adapt to the seven-fold symmetry of the heptameric ClpP, leaving out one of the binding pockets (Figure 2g-h). Due to multivalence, this results in strong, but at the same time flexible binding, which is likely necessary to accommodate the different conformations of ClpX protomers during ATP hydrolysis and substrate processing $12,21,33$.

\section{$\mathrm{N}$-termini of ClpP2 and pore-2 loops of ClpX regulate the entry portal}

ClpX is not only tilted, but also laterally shifted respective to ClpP2 (Figure 3a, d, e). Such an arrangement has also been described for other complexes that display a symmetry mismatch $^{37-39}$. In the case of ClpXP1/2, this results in a misalignment of the central channels of $\mathrm{ClpP}$ and $\mathrm{ClpX}$, creating in a twisted translocation channel with a constriction site at the interface between $\mathrm{ClpP} 2$ and $\mathrm{ClpX}$ (Figure 3d). At this position, the N-terminal loops of $\mathrm{ClpP} 2$ and pore- 2 loops of $\mathrm{ClpX}$ interact with each other. These interactions are expected to be even more dynamic than the flexible contacts mediated by the IGF loops, and coupled to ATP-hydrolysis ${ }^{12,14,40}$. Indeed, the densities corresponding to the $\mathrm{N}$-terminal 
loops of $\mathrm{ClpP} 2$ and pore-2 loops of $\mathrm{ClpX}$ are very weak indicating a higher degree of flexibility in this region of the complex (Supplementary Figures 7,8).

Different conformations of the ClpP N-terminal loops have been previously identified in crystal structures of apo and ADEP-bound $\mathrm{ClpPs}^{11,41,42}$. In the E. coli apo ClpP structure, the N-termini on the apical side of the ClpP barrel are in the "down" conformation, opening one axial pore of the barrel. On the basal side six of the N-termini are in the "up" conformation, with the loops moving out of the axial pore, thereby covering and closing it. It was speculated that the six ClpP N-termini in the "down" conformation would open to match the six-fold symmetry of ClpX and the seventh non-interacting $\mathrm{N}$-terminus would stay in the "down" conformation upon binding to the chaperone. However, in the ADEPbound structure of $E$. coli $\mathrm{ClpP}$ all loops point upwards while they are not resolved in a $B$. subtilis ADEP-bound ClpP structure having made general conclusions difficult so arr $^{41,42}$.

In our cryo-EM structure, residues 6 to 17 are not resolved, but the rest of the density reveals that all seven $\mathrm{N}$-termini of $\mathrm{ClpP} 2$ (the apical side of the barrel facing the chaperone) adopt the "up"-conformation resolving the controversy about their positioning and the accessibility of the pore (Supplementary Fig. 7). The cryo-EM structure demonstrates that the interaction site between the ClpP2 N-termini and the ClpX pore-2 loops is not shielded and freely solvent accessible. In addition, the $\mathrm{N}$-termini undergo a conformational change upon complex formation and adopt the "up" conformation, by which the protein backbone likely gets more solvent exposed and/or flexible. In line with this, deuteration of the ClpP2 Nterminus increased after complex formation (Figure 4, Supplementary Fig. 4). This observation is also supported by reported synchrotron hydroxyl radical footprinting data showing that ClpA binding enhanced the modification rate of an $\mathrm{N}$-terminal peptide of ClpP, pointing towards a higher solvent accessibility ${ }^{43}$.

\section{The C-terminus of ClpP2 shields the hydrophobic groove prior to ClpX binding}

The C-termini of the ClpP2 show two conformations in our structure: a compact conformation that blocks the hydrophobic groove when it does not accommodate an IGF loop, and an extended conformation enlarging the groove when occupied by an IGF loop (Figure 5a). Since the residues of the C-terminus are not conserved (Supplementary Figure 9) and the conformational change is not transmitted to the rest of the protein, an allosteric regulation is rather unlikely. The $\mathrm{C}$-termini probably shield the hydrophobic grooves, when $\mathrm{ClpX}$ is not bound and thereby prevent the interaction with other hydrophobic molecules and increase the stability of the protein in a hydrophilic environment.

To probe this, we deleted the last three to six amino acids of ClpP2. ClpP $1 / 2^{\Delta \mathrm{C}-6}$ precipitated during purification, suggesting that a certain length of the $\mathrm{C}$-terminus is important to protect the hydrophobic groove and facilitate protein stability. $\mathrm{ClpP} 2$ mutants bearing three to five amino acid deletions were however soluble and exhibited a similar peptidolytic activity as the wild type complex (Figure 5b). Interestingly, in protease assays requiring the binding of $\mathrm{ClpX}$, the activity increased with a growing number of amino acid deletions in comparison to the wild type complex (Figure 5c). We interpret this result such that when the C-termini are shorter more complexes are formed because $\mathrm{ClpX}$ can easier access the hydrophobic 
grooves via the IGF-loops. Indeed, in line with this finding the C-termini of most ClpPs which were shown to interact with ClpX are shorter in length (Supplementary Figure 9).

\section{ClpP activation mechanism by ClpX}

Previous crystal structures of $\mathrm{ClpP}$ in its apo-form, i.e. without $\mathrm{ClpX}$ or compound bound, revealed three different conformational states of the protein: "compressed", "compact" and "extended"44-48 (Figure 6). The catalytic triad of the peptidase is only intact in the extended state, suggesting that this is the only active state. ADEPs, that bind to the same site on ClpP as the IGF loops, can induce the transition from the compressed to the extended conformation ${ }^{15}$. In addition, a $\sim 90^{\circ}$ rotation of Tyr63 in the hydrophobic pocket results in the widening of the axial pore by $10-15 \AA$. A mutation of this residue to alanine has the same effect ${ }^{49}$. This "open" extended conformation of ClpP deregulates the protein. Instead of only processing short peptides of five to six residues, it is now capable to degrade large unfolded polypeptides that otherwise could not be processed in the absence of the chaperone (Figure 7) ${ }^{42,43,50}$. It has been speculated that the mechanism of ClpP activation by ClpX would imply similar conformational changes ${ }^{18,49}$.

Our ClpXP1/2 structure demonstrates that this is not the case. ClpP is in the active extended conformation which is very similar to its conformation in the apo-state (Figure 6a, b). Despite the S98A mutation, the catalytic triad is aligned and in its active conformation (Figure 6d, Supplementary Fig. 5b). The ClpP1-P2 heptamers are interconnected via typical interactions of antiparallel $\beta 9$ strands, characteristic for the "extended" active conformation $^{45}$ (Figure 6e). Importantly, the axial pore of ClpP is not widened, when compared to the crystal structure of $B$ subtilis ADEP-bound ClpP (Figure 6c, Supplementary Video 2). A comparison of the interface between the IGF-loop and ADEP with the hydrophobic ClpP pocket reveals that both interact with the same non-polar residues including Ile28, Leu49, Tyr63, Phe83, Ile90, Leu115 (Figure 7a-c). However, binding of ClpX does not induce the rotation of Tyr63 (Figure 7c), which is key to opening the pore. Thus, despite the fact that ADEPs and ClpX share the same binding sites, ClpX does not induce the conformational changes resulting in the opening of ClpP. Instead, binding does not induce any major conformational changes and the diameter of the $\mathrm{ClpP}$ channel is sufficient to accommodate the unfolded peptides that are threaded into the ClpP pore by the chaperone to be processed sequentially within the chamber of the peptidase (Figure 7f).

\section{Discussion}

ClpXP plays a significant role in the production and regulation of bacterial virulence factors during host infection and is therefore considered as a promising target for antimicrobial therapy ${ }^{51,52}$. On the other hand, targeting of the mitochondrial homologues is considered as a novel approach to halt tumor cell proliferation and metastatic competence ${ }^{53}$. Despite the important role of ClpXP in protein degradation, biology and medicine in general, structural knowledge of the dynamic two-component proteolytic machinery has lagged behind. The flexible and dynamic interaction between $\mathrm{ClpX}$ and $\mathrm{ClpP}$ via long flexible IGF- and pore-2 loops, involving a symmetry mismatch, together with the asymmetry of the ClpX ATPase 
make this complex a difficult specimen for structural analysis and probably explain why a high-resolution structure of the complex has been missing so far.

In contrast to previous works, here we utilized the $\mathrm{ClpP} 1 / 2$ heterocomplex from $L$. monocytogenes, showing a higher affinity to $\mathrm{ClpX}$ than the homocomplex. We mutated the proteolytic site and nucleotide binding site of ClpP1/2 and ClpX, respectively, and crosslinked the sample, in order to obtain a ClpXP1/2 complex with superior stability for cryoEM studies. We believe that this was key to determine the ClpXP1/2 structure at an average resolution of $4 \AA$. The resolution for ClpX, however, is lower and therefore does not allow modeling of side chains.

An interesting finding of the current study is the structural visualization of the interface between the hexameric ClpX ATPase and the heptameric ClpP protease, which involves a symmetry mismatch. The structural plasticity, which is necessary for the interaction of the symmetrically different proteins is provided by the flexibility of the IGF-loops. The binding of ClpP to ClpX does not induce major conformational changes of $\mathrm{ClpX}$ and delocalization of distinct AAA+ subunits. The flexibility of ClpP-ClpX interface might be crucial to accommodate different conformations of the ATPase during hydrolysis and proteolysis, and might even allow rotational movement of the ATPase during the repeating cycles of substrate unfolding and translocation. However, further studies are necessary in order to support this scenario.

$\mathrm{ClpX}$ is tilted and slightly shifted relative to ClpP2 and the symmetry-axes of the protease and the ATPase are therefore not aligned. Thus, upon complex formation, the translocation pathway for unfolded peptides is not straight, but twisted. A similar arrangement involving a symmetry mismatch and formation of a twisted peptide translocation channel has been recently described for the PAN-proteasome ${ }^{39}$ and the bacterial $\mathrm{ABC}$ toxin complex ${ }^{37}$. The binding of proteasomal ATPases to the 20S core particle also involves a six-seven symmetry mismatch. However, in this case, the interface is more rigid, since the ATPases bind with their hydrophobic C-termini tightly into pockets at the surface of the $20 \mathrm{~S}$ core particle ("keyin-lock" mechanism) ${ }^{54,55}$. Noteworthy, whereas most of ATPases induce pore opening to allow substrate entry into the proteasomal core, several eukaryotic ATPases (Rpt2, Rpt3 and Rpt5) stably bind to the same pockets of the core particle, but similar to ClpX, do not trigger gate-opening 54,56 .

Surprisingly, although ClpX interacts via the IGF-loops with the same site on ClpP as the potential antibiotic $\mathrm{ADEP}^{17}$, it does not induce the opening of the $\mathrm{ClpP} 1 / 2$ pore, as previously suggested. Thus, the underlying mechanisms of ClpP activation by $\mathrm{ClpX}$ and ADEP are distinct.

Our structure further reveals, that the extended C-terminus of $L$. monocytogenes $\mathrm{ClpP} 1 / 2$ shields the IGF-binding sites prior to $\mathrm{ClpX}$ binding. The length of the $\mathrm{C}$-terminus is apparently crucial to fine-tune the binding affinity to $\mathrm{ClpX}$, among the different species, which might be important for the future design of ClpP-based antibiotics.

The pore-2 loops, that control the peptidase gate and thread the substrate into the ClpP1/2 chamber, are disordered in our structure, underlining the dynamic nature of these 
interactions. However, the overall arrangement of adjacent structural elements suggest that the pore-2 loops are arranged in a spiral-staircase-like manner, similarly to other AAA+ complexes ${ }^{38,57}$.

Interestingly, the ClpXP1/2 complex from L. monocytogenes dimerizes. Only ClpP2 binds to ClpX and two opposing ClpX hexamers dimerize head-to-head through the ZBDs. In contrast, the $E$. coli $\mathrm{ClpP}$ homocomplex is doubly-capped by $\mathrm{ClpX}^{23}$. It is unclear whether the dimerization of the ClpXP1/2 complexes is biologically relevant. The termini of this arrangement of up to four ZBD dimers linking the ClpX hexamers, point directly to their distal pore entries. It is therefore tempting to speculate that this interaction might play a role in substrate binding and even help guiding it into the ClpX pores. Another explanation might be that, at the high concentrations used for EM, two copies of ClpX might recognize each other as substrate. This scenario is however unlikely, because most of ClpX stays intact after incubation of WT ClpX with WT ClpP1/2.

In summary, the cryo-EM structure of ClpXP1/2 provides the necessary basic insights into $\mathrm{ClpXP}$ architecture, essential to understand the molecular mode of action of this dynamic and highly flexible protein degradation machinery. Our results set the stage for future investigations into conformational changes underlying ClpXP ATP hydrolysis and substrate translocation during protein degradation.

\section{Methods}

Cloning

The cloning of pETDuet-1_ClpP1/2 and pET300_ClpX were described previously ${ }^{32}$. ClpX and ClpP1/2 point mutants, $\mathrm{ClpP} 1 / 2^{\Delta \mathrm{C}-3}, \mathrm{ClpP} 1 / 2^{\Delta \mathrm{C}-4}$ and $\mathrm{ClpP} 1 / 2^{\Delta \mathrm{C}-5}$ were generated using the QuikChange ${ }^{\mathrm{TM}}$ technology. For ClpP $1 / 2^{\Delta \mathrm{C}-4}$ and $\mathrm{ClpP} 1 / 2^{\Delta \mathrm{C}-5}$, the pETDuet-1_ClpP1/2 $2^{\Delta \mathrm{C}-3}$ plasmid was used as a template. ClpP1/2 $2^{\Delta \mathrm{C}-6}$ and $\mathrm{ClpX}^{\triangle \mathrm{ZBD}}(\mathrm{E} 183 \mathrm{Q})$ were obtained with primers containing non-overlapping sequences ${ }^{58}$. All primers are listed in Supplementary Table 1

\section{Protein overexpression and purification}

$\mathrm{ClpP} 1 / 2$ and its mutants' variants were overexpressed and purified as follows. The proteins were overexpressed in E. coli BL21(DE3) bearing a pETDuet-1 vector with C-terminally

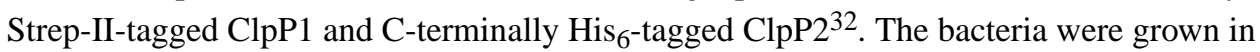
LB medium until $\mathrm{OD}_{600} 0.6$ at $37{ }^{\circ} \mathrm{C}$. Following induction with $1 \mathrm{mM}$ isopropyl- $\beta$-Dthiogalactoside (IPTG), the bacteria were incubated at $37^{\circ} \mathrm{C}$ for $6 \mathrm{~h}$. After harvest, the cells were sonicated on ice in lysis buffer (20 mM MOPS, $300 \mathrm{mM} \mathrm{KCl,} 1 \%$ CHAPS, $10 \%$ glycerol, $\mathrm{pH}$ 7.5) and then kept at room temperature during the rest of the purification. The proteins from the cleared cell lysate were captured in a HisTrap HP $5 \mathrm{ml}$ column (GE Healthcare) in His buffers (20 mM MOPS, $300 \mathrm{mM} \mathrm{KCl,} \mathrm{10 \%} \mathrm{glycerol,} \mathrm{pH} \mathrm{7.5;} \mathrm{+40} \mathrm{mM}$ imidazole for washing) using an ÄKTA Purifier 10 system (GE Healthcare). The proteins were eluted by a $15 \mathrm{~mL}$ gradient from $40 \mathrm{mM}$ to $300 \mathrm{mM}$ imidazole, and the second elution peak was collected. A subsequent chromatography step was carried out on a StrepTrap HP 5 $\mathrm{ml}$ column (GE Healthcare) in Strep buffers (20 mM MOPS, $300 \mathrm{mM} \mathrm{KCl}, 10 \%$ glycerol, 
$\mathrm{pH} 7.5 ;+2.5 \mathrm{mM}$ desthiobiotin for elution). A final gel filtration was performed on a Superdex200 pg 16/60 column (GE Healthcare) in ClpP SEC buffer (20 mM MOPS, 300 $\mathrm{mM} \mathrm{KCl}, 15 \%$ glycerol, $\mathrm{pH}$ 7.0). In the case of the cystein-containing mutants, $1 \mathrm{mM}$ TCEP was added to all buffers.

ClpX(E183Q) and ClpX ${ }^{\Delta Z B D}(\mathrm{E} 183 \mathrm{Q})$ were overexpressed in E. coli BL21(DE3). An expression construct equipped with an $\mathrm{N}$-terminal $\mathrm{His}_{6}$-tag and a TEV cleavage site in pET300 vector was used ${ }^{32}$. The bacteria were grown in $\mathrm{LB}$ medium to $\mathrm{OD}_{600} 0.6$ at $37^{\circ} \mathrm{C}$. After induction with $0.5 \mathrm{mM}$ IPTG, the cells were incubated overnight at $25^{\circ} \mathrm{C}$. After harvest, the cells were resuspended in ClpX lysis buffer (25 mM HEPES, $200 \mathrm{mM} \mathrm{KCl}, 1$ mM DTT, $0.5 \mathrm{mM}$ ATP, $5 \mathrm{mM} \mathrm{MgCl} 2,10 \mathrm{mM}$ imidazole, $5 \%$ glycerol, $\mathrm{pH}$ 7.6) and lysed by ultrasonication. The cleared cell lysate was loaded on a $5 \mathrm{~mL}$ HisTrap HP column (GE Healthcare). The column was washed with ClpX wash buffer (25 mM HEPES, $200 \mathrm{mM}$ $\mathrm{KCl}, 1 \mathrm{mM}$ DTT, 5\% glycerol, $40 \mathrm{mM}$ imidazole, $\mathrm{pH}$ 7.6). The protein was eluted with ClpX elution buffer (25 mM HEPES, $200 \mathrm{mM} \mathrm{KCl,} 1 \mathrm{mM}$ DTT, 5\% glycerol, $300 \mathrm{mM}$ imidazole, $\mathrm{pH}$ 7.6). The protein fractions were pooled, $1 \mathrm{mM}$ EDTA and TEV protease [1.25 $\mathrm{mg}$ for $\mathrm{ClpX}(\mathrm{E} 183 \mathrm{Q})$ and $3.75 \mathrm{mg}$ for $\left.\mathrm{ClpX}^{\Delta \mathrm{ZBD}}(\mathrm{E} 183 \mathrm{Q})\right]$ were added and the reaction mixture was incubated at $10^{\circ} \mathrm{C}$ overnight. Complete TEV cleavage was verified by intactprotein mass-spectrometry. The protein solution was loaded on a Superdex200 pg 16/60 column (GE Healthcare) and eluted in ClpX SEC buffer (25 mM HEPES, $200 \mathrm{mM} \mathrm{KCl}, 1$ mM DTT, $0.5 \mathrm{mM}$ ATP, $5 \mathrm{mM} \mathrm{MgCl} 2,5 \%$ glycerol, pH 7.6). ClpX(WT), ClpX(V264C), $\mathrm{ClpX}(\mathrm{I} 265 \mathrm{C}), \mathrm{ClpX}(\mathrm{G} 266 \mathrm{C})$ and $\mathrm{ClpX}(\mathrm{F} 267 \mathrm{C})$ were overexpressed and purified similarly with the following modifications: the buffers contained $1 \mathrm{mM}$ TCEP instead of DTT, and the ClpX wash buffer and ClpX elution buffer contained additionally $0.5 \mathrm{mM}$ ATP and $5 \mathrm{mM}$ $\mathrm{MgCl}_{2}$. The TEV digestion step was omitted.

N-terminally Strep-II-tagged eGFP with a C-terminal SsrA tag (AGKEKQNLAFAA) was overexpressed in E. coli SG1146a ( $\triangle c l p P)$ using pET55-Dest expression vector and purified by affinity chromatography and gel filtration as described previously 15,32 .

Creatine kinase (product no. 10127566 001), lactate dehydrogenase (product no. 10128 155001 ) and pyruvate kinase (product no. 10127876 001) were purchased from Roche.

\section{Isolation of the CIpXP complex}

$4.4 \mathrm{nmol}(\mathrm{ClpP} 1 / 2)_{14}$ and $3.3 \mathrm{nmol} \mathrm{ClpX} 6$ were incubated for $10 \mathrm{~min}$ at $37{ }^{\circ} \mathrm{C}$ in PZA buffer (25 mM HEPES, $200 \mathrm{mM} \mathrm{KCl,} 5 \mathrm{mM} \mathrm{MgCl} 2,1 \mathrm{mM}$ DTT, $0.5 \mathrm{mM}$ ATP, 15\% glycerol, $\mathrm{pH}$ 7.6). The samples were loaded onto a Superose 6 increase 10/300 column (GE Healthcare) connected to an ÄKTA Purifier 10 system (GE Healthcare) and eluted at $0.2 \mathrm{~mL} / \mathrm{min}$ flow rate. Samples were taken at $12 \mathrm{~mL}$ retention volume for EM and HDX-MS measurements. For cryoEM, the sample was diluted 1:3 with glycerol-free PZA buffer and 0.1\% glutaraldehyde was added. The reaction was quenched after $30 \mathrm{~s}$ with 2 eq. Tris-HCl. For SDS-PAGE, $4.4 \mu \mathrm{g}$ protein was loaded on a gel and stained with Coomassie blue after separation. 


\section{Electron microscopy}

Sample quality was examined by negative stain EM. Sample from the respective fraction was further diluted to a concentration of $0.01-0.03 \mathrm{mg} \mathrm{ml}^{-1}$ and negative stain EM was performed as described previously ${ }^{59}$. Images were recorded with a JEOL JEM-1400 equipped with a $4 \mathrm{~K}$ CMOS detector F416 (TVIPS) at a pixel size of $1.84 \AA$ A. For cryoEM, 4 $\mu \mathrm{l}$ of cross-linked ClpXP1/2 dimers at a concentration of $0.045 \mathrm{mg} \mathrm{ml}^{-1}$ were applied to a glow-discharged quantifoil $2 / 1 \mathrm{Cu}$ grid with an additional $2 \mathrm{~nm}$ thin carbon layer and after an incubation time of $45 \mathrm{sec}$, rapidly plunge-frozen using a CryoPlunge3 (Cp3, Gatan) at 90\% humidity. To improve ice quality and thickness distribution, $0.01 \%$ Tween-20 was added shortly prior plunging. The quality of the grids was screened with a JEOL JEM 1400 and a FEI Tecnai Spirit, both equipped with a $\mathrm{LaB}_{6}$ cathode and a $4 \mathrm{~K}$ CMOS detector F416 (TVIPS). A cryoEM dataset was acquired on a FEI Titan KRIOS at $300 \mathrm{kV}$ equipped with spherical aberration corrector and a Falcon III direct detector (linear mode) at a x112,807 magnification (x59,000 nominal magnification), corresponding to a pixel size of $1.1 \AA$. Each exposure was recorded with a total dose of $\sim 114$ electrons $/ \AA^{2}$ and a total exposure time of 2 $\mathrm{sec}$ (frame rate of $50 \mathrm{msec}$ ). A total of 3200 micrographs were collected using the EPU software (FEI).

\section{Image processing and reconstruction}

The frames were aligned, averaged and dose-weighted using unblur and sum_movie ${ }^{60}$. Unweighted full-dose images were further used to estimate the CTF parameters using CTER 61 (SPHIRE) ${ }^{25}$. Dose weighted full-dose images were used for all other steps of image processing. ClpXP1/2 dimers were picked automatically using EMAN2's ${ }^{62}$ neuralnet e2boxer. Further data processing was performed using the software package SPHIRE $^{25}$. After inspection of micrographs using the CTF-assessment-GUI, 273,300 single particles were selected for further processing. The particle stack was subjected to 2D-clustering using ISAC2 (SPHIRE), resulting in a "clean" stack of 143,901 single particles producing stable and reproducible 2D-class averages. The 2D class-averages were used to calculate a 3D volume, using VIPER. After masking, this volume was used as the reference for a 3D refinement using Meridien (SPHIRE), which resulted in a 13 Å density map, as estimated by the "gold-standard" FSC. In agreement to the 2D clustering results (Supplementary Video 1), further 3D clustering using Sort3D (SPHIRE) confirmed that the ClpXP1/2 dimer is a continuously flexible structure (Supplementary Figure 1g). Independent refinement of the resulting subsets did not, however, further improve the resolution of the volume.

We then manually picked the ClpXP1/2 monomers within each ClpXP1/2-dimer for 10 representative micrographs of the dataset and used these data to train $\mathrm{crYOLO}^{63}$, which then automatically selected 613,322 single particles. After 2D and 3D clustering, a final "clean" stack of 383.927 particles was used for further refinement. During the first rounds of the refinement, we applied local symmetrization of the reference after each refinement round, as previously described ${ }^{64,65}$ i.e. after each refinement round the density of $\mathrm{ClpP}$ was symmetrized using $D 7$ symmetry, whereas the density of $\mathrm{ClpX}$ was scaled in order to put an additional weight on this region during the asymmetric refinement. Finally, both densities (ClpX and ClpP) were combined and the resulting volume was used as a reference for the subsequent refinement iteration. This procedure was performed during the initial rounds in 
order to obtain global projection parameters. The user function was not applied during the local refinements. This resulted in a density map with an average resolution of $4 \AA$, where the resolution of the density decreases towards ClpX (Supplementary Figure 2). The average resolution was calculated between two independently refined "half maps" at the 0.143 FSC criterion. The estimated accuracy of rotation and translation search during the last refinement round was estimated to $1.78^{\circ}$ and 1.02 pixels, respectively. Local resolution was computed using the "Local Resolution" tool in SPHIRE. 3D clustering into four groups was performed using the RSORT3D tool of SPHIRE. However, according to the ANOVA analysis, the resulting volumes were not reproducible and were therefore not considered for further analysis. 3D Refinement and Clustering focusing on the density of ClpX, after removing the $\mathrm{ClpP}$ signal from the dataset, did also not result into further improvement of the ClpX density. The density of ClpP was auto-sharpened locally using phenix.auto_sharpen 66 and filtered to its average resolution of 3.9 $\AA$. The ClpX desnity was filtered to an average resolution of $6.5 \AA$ and sharpened with an ad-hoc b-factor of $-240 \AA^{2}$. Angular distribution plots were computed using SPHIRE. Sharpened 2D class averages were computed with 3500 members per group.

\section{Atomic modelling}

We built a homology model of ClpX with SWISS-MODEL ${ }^{67}$ using ADP-bound E. coli ClpX (PDB-ID 3HWS, Chain A) and ATP $\gamma$ S-bound E. coli ClpX (PDB-ID 4I81, Chain B). We then used UCSF Chimera ${ }^{68}$ to fit the structures of ClpX's homology model and ClpP1/2 (PDBID 4RYF ${ }^{32}$ into the cryo-EM density. We used the RosettaES protocol ${ }^{69}$ to build the missing residues 9-16 for each ClpP2 subunit. Residues 1-2 were manually built in $\operatorname{Coot}^{70}$.

With the complete model, we performed several iterative runs of molecular dynamics flexible fitting (MDFF) ${ }^{71}$ and manual adjustment with Coot, paying particular attention to the fitting of the IGF loops. In the initial run, we applied 6-fold symmetry to ClpX, allowing regions poorly supported by the density to settle into reasonable conformations. This restraint was later removed. For the final iterations, we also included a step of real-space refinement in Phenix ${ }^{72}$, to decrease the number of Ramachandran outliers and to fit the atomic B-factors.

The necessary files for the MDFF runs were set up with $\mathrm{VMD}^{73}$ and all simulations were performed in $\mathrm{NAMD}^{74}$, using the CHARMM $36 \mathrm{~m}$ force field ${ }^{75}$ with the implicit solvation model implemented in NAMD.

For the proper modeling of the structure with MDFF, we included all missing regions of the structures, even if their density does not allow full atomic modeling. After refinement, we removed all those from the final model. The quality of this model was assessed in Phenix, using the Molprobity ${ }^{76}$ and EMRinger scores ${ }^{77}$ as well as the overall geometry of the structure.

Sequence conservation was analyzed using the ConSurfserver ${ }^{78}$. Analysis of the channel pathway was performed with $\mathrm{ChExVis}^{79}$. Electron density maps and models were visualized using Chimera ${ }^{69}$ and Chimera $\mathrm{X}^{80}$. 


\section{Peptidase assay}

In this assay, the degradation of a fluorogenic tripeptide was measured, for which $\mathrm{ClpX}$ was not required. $99 \mu \mathrm{L} 1 \mu \mathrm{M} \mathrm{ClpP1/2}$ was incubated in PZ buffer (25 mM HEPES, $200 \mathrm{mM}$ $\mathrm{KCl}, 5 \mathrm{mM} \mathrm{MgCl} 2,1 \mathrm{mM}$ DTT, $10 \%$ glycerol, $\mathrm{pH}$ 7.6) in flat bottom black 96-well plates for $15 \mathrm{~min}$ at $30^{\circ} \mathrm{C} .1 \mu \mathrm{L}$ acetylalanyl-homoarginyl-2-aminooctanoyl-7-amino-4carbamoylmethylcoumarin (Ac-Ala-hArg-2-Aoc-ACC) substrate (10 mM stock in DMSO) was added and the fluorescence was measured $(380 \mathrm{~nm}, 430 \mathrm{~nm})$ with an infinite M200Pro plate reader (Tecan) at $30{ }^{\circ} \mathrm{C}$. Data were recorded in triplicate and two independent experiments were performed. Peptidase activity was determined by linear regression using Microsoft Excel and plots were made with GraphPad Prism 6.

\section{Protease assay}

Protease assays were carried out in flat bottom white 96-well plates in a final volume of 60 $\mu \mathrm{L}$. $(\mathrm{ClpP} 1 / 2)_{14}(0.2 \mu \mathrm{M}), \mathrm{ClpX}_{6}(0.4 \mu \mathrm{M})$ and ATP regeneration mix (4 mM ATP, $16 \mathrm{mM}$ creatine phosphate, $20 \mathrm{U} / \mathrm{mL}$ creatine kinase) were pre-incubated for $15 \mathrm{~min}$ at $30{ }^{\circ} \mathrm{C}$ in $\mathrm{PZ}$ buffer. $0.8 \mu \mathrm{M}$ eGFP-SsrA substrate was added and fluorescence was measured $(485 \mathrm{~nm}$, $535 \mathrm{~nm}$ ) at $30^{\circ} \mathrm{C}$. Data were recorded in triplicate and at least two independent experiments were performed. Protease activity was determined by linear regression using Microsoft Excel and plots were made with GraphPad Prism 6.

\section{ATPase assay}

$90 \mu \mathrm{L} 2 \mu \mathrm{M}$ ClpX in ATPase buffer (100 mM HEPES, $200 \mathrm{mM} \mathrm{KCl}, 20 \mathrm{mM} \mathrm{MgCl}_{2}, 1 \mathrm{mM}$ DTT, $1 \mathrm{mM}$ NADH, $2 \mathrm{mM}$ phosphoenolpyruvate, $50 \mathrm{U} / \mathrm{mL}$ lactate dehydrogenase, $50 \mathrm{U} / \mathrm{mL}$ pyruvate kinase, $5 \%$ glycerol, $\mathrm{pH} 7.5$ ) was added to a flat bottom transparent 96-well plate and incubated for $15 \mathrm{~min}$ at $37^{\circ} \mathrm{C}$. The reaction was started by the addition of $10 \mu \mathrm{L} 200$ $\mathrm{mM}$ ATP in $100 \mathrm{mM}$ HEPES, $\mathrm{pH} 7.5$. Absorption at $340 \mathrm{~nm}$ was measured at $37^{\circ} \mathrm{C}$. Two independent experiments with three replicates each were carried out. ATPase activity was determined by linear regression using Microsoft Excel after subtraction of the background signal (measurement without ClpX), and the plot was made with GraphPad Prism 6.

\section{Hydrogen/deuterium exchange mass-spectrometry (HDX-MS)}

HDX-MS experiments were performed using an ACQUITY UPLC M-class system equipped with automated HDX technology (Waters). HDX kinetics were determined by taking data points at $0,10,60,600,1800$ and $7200 \mathrm{~s}$ at $20^{\circ} \mathrm{C}$. At each data point of the kinetic, $3 \mu \mathrm{L}$ of a solution of $30 \mu \mathrm{M}$,free" ClpP1/2 and ,free" ClpX were analyzed and compared to the $(\mathrm{ClpXP} 1 / 2)_{2}$ complex $(1.4 \mu \mathrm{M})$. The respective protein solutions were diluted automatically 1:20 into 99.9\% $\mathrm{D}_{2} \mathrm{O}$-containing buffer (25 mM HEPES, $200 \mathrm{mM} \mathrm{KCl}, 5 \mathrm{mM} \mathrm{MgCl} 2,0.5$ $\mathrm{mM}$ ATP, $1 \mathrm{mM}$ TCEP, $5 \%$ glycerol, $\mathrm{pH}$ 7.6). As reference, all samples were analyzed in $\mathrm{H}_{2} \mathrm{O}$-containing buffers. The reaction mixture was quenched by the addition of 1:1 $200 \mathrm{mM}$ $\mathrm{KH}_{2} \mathrm{PO}_{4}, 200 \mathrm{mM} \mathrm{Na}_{2} \mathrm{HPO}_{4}, \mathrm{pH} 2.3$ (titrated with $\mathrm{HCl}$ ) at $1{ }^{\circ} \mathrm{C}$ and $50 \mu \mathrm{L}$ of the resulting sample were subjected to on-column peptic digest on a Waters Enzymate BEH pepsin column $2.1 \times 30 \mathrm{~mm}$ at $20{ }^{\circ} \mathrm{C}$. Peptides were separated by reverse phase chromatography at $0{ }^{\circ} \mathrm{C}$ in trapping mode using a Waters Acquity UPLC C18 $1.7 \mu \mathrm{m}$ Vangard $2.1 \times 5 \mathrm{~mm}$ precolumn and a Waters Aquity UPLC BEH C18 $1.7 \mu \mathrm{m} 1 \times 100 \mathrm{~mm}$ separation column. For 
separation, a gradient increasing the acetonitrile concentration stepwise from 5 to $35 \%$ in 6 min, from 35 to $40 \%$ in $1 \mathrm{~min}$ and from 40 to $95 \%$ in $1 \mathrm{~min}$ was applied and the eluted peptides were analyzed using an in-line Synapt G2-S QTOF HDMS mass spectrometer (Waters). UPLC was performed in protonated solvents ( $0.1 \%$ formic acid), allowing deuterium to be replaced with hydrogen from side chains and amino/carboxyl termini that exchange much faster than backbone amide linkages ${ }^{81}$. All experiments were performed in duplicate. Deuterium levels were not corrected for back exchange and are therefore reported as relative deuterium levels ${ }^{82}$. The use of an automated system, i.e. handling all samples at identical conditions, negotiates the need for back exchange correction. MS data were collected over an m/z range of 100-2000, and are available online as source data [AU: correct?]. Mass accuracy was ensured by calibration with Glu-fibrino peptide B (Waters) and peptides were identified by triplicates MSE ramping the collision energy from 20-50 V. MS data were analyzed with the PLGS 3.0.3 and DynamX 3.0 software packages and all spectra were checked manually. For each peptide, relative uptake values were determined as follows: relative uptake $[\%]=$ deuterium uptake $\times 100 /$ maximal uptake. For each amino acid, the average of the relative uptake of all peptides covering the amino acid was calculated. The difference of the relative deuterium uptake between the "free" and "complex" states was calculated for each amino acid. Data were analyzed and visualized using custom MATLAB and python scripts, UCSF Chimera 1.12 and OriginPro 2016.

\section{Supplementary Material}

Refer to Web version on PubMed Central for supplementary material.

\section{Acknowledgements}

We thank O. Hofnagel for assistance in electron microscopy and Dr. M. Lakemeyer for building ClpX homology models. We are grateful to Dr. M. Haslbeck, G. M. Feind and F. Rührnößl for HDX-MS measurements. This work was supported by the Max Planck Society (to S.R.), the European Research Council (FP7/2007-2013) (grant no. 615984) (to S.R.) and the Deutsche Forschungsgemeinschaft (SFB1035) (to S.A.S).

\section{References}

1. Bhandari V, et al. The Role of ClpP Protease in Bacterial Pathogenesis and Human Diseases. ACS Chem Biol. 2018; 13:1413-1425. [PubMed: 29775273]

2. Gaillot O, Pellegrini E, Bregenholt S, Nair S, Berche P. The ClpP serine protease is essential for the intracellular parasitism and virulence of Listeria monocytogenes. Mol Microbiol. 2000; 35:12861294. [PubMed: 10760131]

3. Yu AYH, Houry WA. ClpP: a distinctive family of cylindrical energy-dependent serine proteases. FEBS Lett. 2007; 581:3749-3757. [PubMed: 17499722]

4. Brötz-Oesterhelt $\mathrm{H}$, et al. Dysregulation of bacterial proteolytic machinery by a new class of antibiotics. Nat Med. 2005; 11:1082-1087. [PubMed: 16200071]

5. Sauer RT, Baker TA. AAA+ proteases: ATP-fueled machines of protein destruction. Annu Rev Biochem. 2011; 80:587-612. [PubMed: 21469952]

6. Beuron F, et al. At sixes and sevens: characterization of the symmetry mismatch of the ClpAP chaperone-assisted protease. Journal of Structural Biology. 1998; 123:248-259. [PubMed: 9878579]

7. Grimaud R, Kessel M, Beuron F, Steven AC, Maurizi MR. Enzymatic and structural similarities between the Escherichia coli ATP-dependent proteases, ClpXP and ClpAP. Journal of Biological Chemistry. 1998; 273:12476-12481. [PubMed: 9575205] 
8. Wang J, Hartling JA, Flanagan JM. The structure of ClpP at 2.3 A resolution suggests a model for ATP-dependent proteolysis. Cell. 1997; 91:447-456. [PubMed: 9390554]

9. Banecki B, Wawrzynow A, Puzewicz J, Georgopoulos C, Zylicz M. Structure-function analysis of the zinc-binding region of the Clpx molecular chaperone. Journal of Biological Chemistry. 2001; 276:18843-18848. [PubMed: 11278349]

10. Baker TA, Sauer RT. ClpXP, an ATP-powered unfolding and protein-degradation machine. Biochim Biophys Acta. 2012; 1823:15-28. [PubMed: 21736903]

11. Bewley MC, Graziano V, Griffin K, Flanagan JM. The asymmetry in the mature amino-terminus of ClpP facilitates a local symmetry match in ClpAP and ClpXP complexes. Journal of Structural Biology. 2006; 153:113-128. [PubMed: 16406682]

12. Joshi SA, Hersch GL, Baker TA, Sauer RT. Communication between ClpX and ClpP during substrate processing and degradation. Nat Struct Mol Biol. 2004; 11:404-411. [PubMed: 15064753]

13. Martin A, Baker TA, Sauer RT. Distinct static and dynamic interactions control ATPase-peptidase communication in a AAA+ protease. Mol Cell. 2007; 27:41-52. [PubMed: 17612489]

14. Kim YI, et al. Molecular determinants of complex formation between Clp/Hsp100 ATPases and the ClpP peptidase. Nat Struct Biol. 2001; 8:230-233. [PubMed: 11224567]

15. Gersch M, et al. AAA+ chaperones and acyldepsipeptides activate the ClpP protease via conformational control. Nat Commun. 2015; 6:6320. [PubMed: 25695750]

16. Kirstein J, et al. The antibiotic ADEP reprogrammes ClpP, switching it from a regulated to an uncontrolled protease. EMBO Mol Med. 2009; 1:37-49. [PubMed: 20049702]

17. Schmitz KR, Carney DW, Sello JK, Sauer RT. Crystal structure of Mycobacterium tuberculosis ClpP1P2 suggests a model for peptidase activation by AAA+ partner binding and substrate delivery. Proc Natl Acad Sci USA. 2014; 111:E4587-95. [PubMed: 25267638]

18. Alexopoulos J, et al. Structural determinants stabilizing the axial channel of ClpP for substrate translocation. Mol Microbiol. 2013; 90:167-180. [PubMed: 23927726]

19. Zeiler E, et al. Vibralactone as a tool to study the activity and structure of the ClpP1P2 complex from Listeria monocytogenes. Angew Chem Int Ed Engl. 2011; 50:11001-11004. [PubMed: 21954175]

20. Balogh D, et al. Insights into ClpXP proteolysis: heterooligomerization and partial deactivation enhance chaperone affinity and substrate turnover in Listeria monocytogenes. Chem Sci. 2017; 8:1592-1600. [PubMed: 28451288]

21. Amor AJ, Schmitz KR, Sello JK, Baker TA, Sauer RT. Highly Dynamic Interactions Maintain Kinetic Stability of the ClpXP Protease During the ATP-Fueled Mechanical Cycle. ACS Chem Biol. 2016; 11:1552-1560. [PubMed: 27003103]

22. Hersch GL, Burton RE, Bolon DN, Baker TA, Sauer RT. Asymmetric interactions of ATP with the AAA+ ClpX6 unfoldase: allosteric control of a protein machine. Cell. 2005; 121:1017-1027. [PubMed: 15989952]

23. Ortega J, Singh SK, Ishikawa T, Maurizi MR, Steven AC. Visualization of substrate binding and translocation by the ATP-dependent protease, ClpXP. Mol Cell. 2000; 6:1515-1521. [PubMed: 11163224]

24. Wagner T, et al. SPHIRE-crYOLO is a fast and accurate fully automated particle picker for cryoEM. Communications Biology. 2019; 2:218. [PubMed: 31240256]

25. Moriya T, et al. High-resolution Single Particle Analysis from Electron Cryo-microscopy Images Using SPHIRE. J Vis Exp. 2017; doi: 10.3791/55448

26. Donaldson LW, Wojtyra U, Houry WA. Solution structure of the dimeric zinc binding domain of the chaperone ClpX. Journal of Biological Chemistry. 2003; 278:48991-48996. [PubMed: 14525985]

27. Wojtyra UA, Thibault G, Tuite A, Houry WA. The N-terminal zinc binding domain of ClpX is a dimerization domain that modulates the chaperone function. Journal of Biological Chemistry. 2003; 278:48981-48990. [PubMed: 12937164]

28. Stinson BM, et al. Nucleotide binding and conformational switching in the hexameric ring of a AAA+ machine. Cell. 2013; 153:628-639. [PubMed: 23622246] 
29. Trabuco LG, Villa E, Mitra K, Frank J, Schulten K. Flexible fitting of atomic structures into electron microscopy maps using molecular dynamics. Structure/Folding and Design. 2008; 16:673-683. [PubMed: 18462672]

30. Fux A, Korotkov VS, Schneider M, Antes I, Sieber SA. Chemical Cross-Linking Enables Drafting ClpXP Proximity Maps and Taking Snapshots of In Situ Interaction Networks. Cell Chem Biol. 2018; doi: 10.1016/j.chembiol.2018.10.007

31. Leodolter J, Warweg J, Weber-Ban E. The Mycobacterium tuberculosis ClpP1P2 Protease Interacts Asymmetrically with Its ATPase Partners ClpX and ClpC1. PLOS ONE. 2015; 10:e125345. [PubMed: 25933022]

32. Dahmen M, Vielberg M-T, Groll M, Sieber SA. Structure and mechanism of the caseinolytic protease ClpP1/2 heterocomplex from Listeria monocytogenes. Angew Chem Int Ed Engl. 2015; 54:3598-3602. [PubMed: 25630955]

33. Glynn SE, Martin A, Nager AR, Baker TA, Sauer RT. Structures of asymmetric ClpX hexamers reveal nucleotide-dependent motions in a AAA+ protein-unfolding machine. Cell. 2009; 139:744756. [PubMed: 19914167]

34. Gates SN, et al. Ratchet-like polypeptide translocation mechanism of the AAA+ disaggregase Hsp104. Science. 2017; 357:273-279. [PubMed: 28619716]

35. Lo Y-H, et al. Cryo-EM structure of the essential ribosome assembly AAA-ATPase Rix7. Nat Commun. 2019; 10:513. [PubMed: 30705282]

36. Ripstein ZA, Huang R, Augustyniak R, Kay LE, Rubinstein JL. Structure of a AAA+ unfoldase in the process of unfolding substrate. Elife. $2017 ; 6: 43$.

37. Gatsogiannis $C$, et al. Tc toxin activation requires unfolding and refolding of a $\beta$-propeller. Nature. 2018; 9:1.

38. de la Peña AH, Goodall EA, Gates SN, Lander GC, Martin A. Substrate-engaged 26S proteasome structures reveal mechanisms for ATP-hydrolysis-driven translocation. Science. 2018; 362

39. Majumder P, et al. Cryo-EM structures of the archaeal PAN-proteasome reveal an around-the-ring ATPase cycle. Proc Natl Acad Sci USA. 2019; 116:534-539. [PubMed: 30559193]

40. Martin A, Baker TA, Sauer RT. Diverse pore loops of the AAA+ ClpX machine mediate unassisted and adaptor-dependent recognition of ssrA-tagged substrates. Mol Cell. 2008; 29:441-450. [PubMed: 18313382]

41. Lee B-G, et al. Structures of ClpP in complex with acyldepsipeptide antibiotics reveal its activation mechanism. Nature Publishing Group. 2010; 17:471-478.

42. Li DHS, et al. Acyldepsipeptide antibiotics induce the formation of a structured axial channel in ClpP: A model for the ClpX/ClpA-bound state of ClpP. Chem Biol. 2010; 17:959-969. [PubMed: 20851345]

43. Jennings LD, Bohon J, Chance MR, Licht S. The ClpP N-terminus coordinates substrate access with protease active site reactivity. Biochemistry. 2008; 47:11031-11040. [PubMed: 18816064]

44. Geiger SR, Böttcher T, Sieber SA, Cramer P. A conformational switch underlies ClpP protease function. Angew Chem Int Ed Engl. 2011; 50:5749-5752. [PubMed: 21544912]

45. Gersch M, List A, Groll M, Sieber SA. Insights into structural network responsible for oligomerization and activity of bacterial virulence regulator caseinolytic protease $\mathrm{P}(\mathrm{ClpP})$ protein. J Biol Chem. 2012; 287:9484-9494. [PubMed: 22291011]

46. Kimber MS, et al. Structural and theoretical studies indicate that the cylindrical protease ClpP samples extended and compact conformations. Structure. 2010; 18:798-808. [PubMed: 20637416]

47. Ye F, et al. Helix unfolding/refolding characterizes the functional dynamics of Staphylococcus aureus Clp protease. J Biol Chem. 2013; 288:17643-17653. [PubMed: 23625918]

48. Zhang J, et al. Structural switching of Staphylococcus aureus Clp protease: a key to understanding protease dynamics. J Biol Chem. 2011; 286:37590-37601. [PubMed: 21900233]

49. Ni T, et al. Characterization of Gain-of-Function Mutant Provides New Insights into ClpP Structure. ACS Chem Biol. 2016; 11:1964-1972. [PubMed: 27171654]

50. Stahl M, Sieber SA. An amino acid domino effect orchestrates ClpP's conformational states. Curr Opin Chem Biol. 2017; 40:102-110. [PubMed: 28910721] 
51. Böttcher T, Sieber SA. Beta-lactones as privileged structures for the active-site labeling of versatile bacterial enzyme classes. Angew Chem Int Ed Engl. 2008; 47:4600-4603. [PubMed: 18383487]

52. Frees D, et al. Clp ATPases are required for stress tolerance, intracellular replication and biofilm formation in Staphylococcus aureus. Mol Microbiol. 2004; 54:1445-1462. [PubMed: 15554981]

53. Cole A, et al. Inhibition of the Mitochondrial Protease ClpP as a Therapeutic Strategy for Human Acute Myeloid Leukemia. Cancer Cell. 2015; 27:864-876. [PubMed: 26058080]

54. Eisele MR, et al. Expanded Coverage of the 26S Proteasome Conformational Landscape Reveals Mechanisms of Peptidase Gating. Cell Rep. 2018; 24:1301-1315.e5. [PubMed: 30067984]

55. Smith DM, et al. Docking of the proteasomal ATPases' carboxyl termini in the 20S proteasome's alpha ring opens the gate for substrate entry. Mol Cell. 2007; 27:731-744. [PubMed: 17803938]

56. Wehmer M, et al. Structural insights into the functional cycle of the ATPase module of the $26 \mathrm{~S}$ proteasome. Proc Natl Acad Sci USA. 2017; 114:1305-1310. [PubMed: 28115689]

57. Deville C, Franke K, Mogk A, Bukau B, Saibil HR. Two-Step Activation Mechanism of the ClpB Disaggregase for Sequential Substrate Threading by the Main ATPase Motor. Cell Rep. 2019; 27:3433-3446.e4. [PubMed: 31216466]

58. Liu H, Naismith JH. An efficient one-step site-directed deletion, insertion, single and multiple-site plasmid mutagenesis protocol. BMC Biotechnol. 2008; 8:91. [PubMed: 19055817]

59. Gatsogiannis C, et al. Membrane insertion of a Tc toxin in near-atomic detail. Nature Publishing Group. 2016; doi: 10.1038/nsmb.3281

60. Grant T, Grigorieff N. Measuring the optimal exposure for single particle cryo-EM using a $2.6 \AA$ reconstruction of rotavirus VP6. Elife. 2015; 4:e06980. [PubMed: 26023829]

61. Penczek PA, et al. CTER-rapid estimation of CTF parameters with error assessment. Ultramicroscopy. 2014; 140:9-19. [PubMed: 24562077]

62. Tang G, et al. EMAN2: An extensible image processing suite for electron microscopy. Journal of Structural Biology. 2007; 157:38-46. [PubMed: 16859925]

63. Wagner T, et al. SPHIRE-crYOLO: A fast and well-centering automated particle picker for cryoEM. bioRxiv. 2018; doi: 10.1101/356584

64. Tanaka Y, et al. Cryo-EM reveals the asymmetric assembly of squid hemocyanin. IUCrJ. 2019; 6:426-437.

65. Gatsogiannis $\mathrm{C}$, et al. Tc toxin activation requires unfolding and refolding of a $\beta$-propeller. Nature. 2018; :1-5. DOI: 10.1038/s41586-018-0556-6

66. Terwilliger TC, Sobolev OV, Afonine PV, Adams PD. Automated map sharpening by maximization of detail and connectivity. Acta Crystallogr D Struct Biol. 2018; 74:545-559. [PubMed: 29872005]

67. Biasini M, et al. SWISS-MODEL: modelling protein tertiary and quaternary structure using evolutionary information. Nucleic Acids Res. 2014; 42:W252-8. [PubMed: 24782522]

68. Pettersen EF, et al. UCSF Chimera?A visualization system for exploratory research and analysis. J Comput Chem. 2004; 25:1605-1612. [PubMed: 15264254]

69. Frenz B, Walls AC, Egelman EH, Veesler D, DiMaio F. RosettaES: a sampling strategy enabling automated interpretation of difficult cryo-EM maps. Nature Methods. 2017; 14:797-800. [PubMed: 28628127]

70. Emsley P, Lohkamp B, Scott WG, Cowtan K. Features and development of Coot. Acta Crystallogr D Biol Crystallogr. 2010; 66:486-501. [PubMed: 20383002]

71. Singharoy A, et al. Molecular dynamics-based refinement and validation for sub-5 A cryo-electron microscopy maps. Elife. 2016; 5:213.

72. Adams PD, et al. PHENIX: a comprehensive Python-based system for macromolecular structure solution. Acta Crystallogr D Biol Crystallogr. 2010; 66:213-221. [PubMed: 20124702]

73. Humphrey W, Dalke A, Schulten K. VMD: visual molecular dynamics. J Mol Graph. 1996; 14:338-27-8. [PubMed: 8744570]

74. Phillips JC, et al. Scalable molecular dynamics with NAMD. J Comput Chem. 2005; 26:17811802. [PubMed: 16222654]

75. Huang J, et al. CHARMM36m: an improved force field for folded and intrinsically disordered proteins. Nature Methods. 2017; 14:71-73. [PubMed: 27819658] 
76. Chen VB, et al. MolProbity: all-atom structure validation for macromolecular crystallography. Acta Crystallogr D Biol Crystallogr. 2010; 66:12-21. [PubMed: 20057044]

77. Barad BA, et al. EMRinger: side chain-directed model and map validation for 3D cryo-electron microscopy. Nature Methods. 2015; 12:943-946. [PubMed: 26280328]

78. Ashkenazy H, Erez E, Martz E, Pupko T, Ben-Tal N. ConSurf 2010: calculating evolutionary conservation in sequence and structure of proteins and nucleic acids. Nucleic Acids Res. 2010; 38:W529-33. [PubMed: 20478830]

79. Masood TB, Sandhya S, Chandra N, Natarajan V. CHEXVIS: a tool for molecular channel extraction and visualization. BMC Bioinformatics. 2015; 16:119. [PubMed: 25888118]

80. Goddard TD, et al. UCSF ChimeraX: Meeting modern challenges in visualization and analysis. Protein Sci. 2018; 27:14-25. [PubMed: 28710774]

81. Englander SW, Kallenbach NR. Hydrogen exchange and structural dynamics of proteins and nucleic acids. Q Rev Biophys. 1983; 16:521-655. [PubMed: 6204354]

82. Wales TE, Engen JR. Hydrogen exchange mass spectrometry for the analysis of protein dynamics. Mass Spectrom Rev. 2006; 25:158-170. [PubMed: 16208684] 

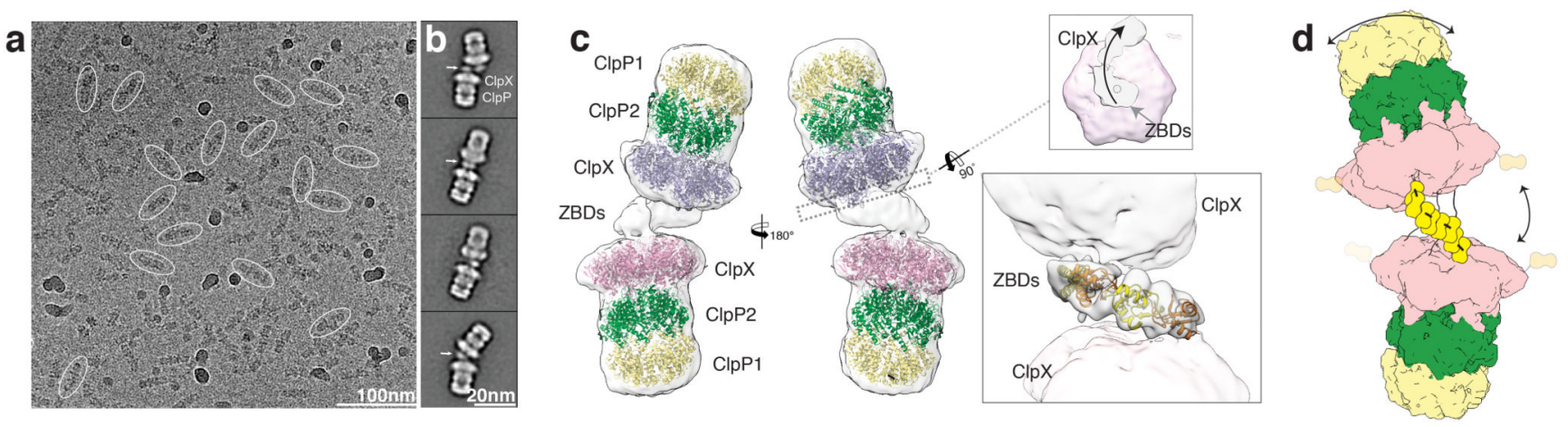

Figure 1. LmClpXP1/2 forms flexible dimers via the ZBDs.

a) Typical low-dose cryo-EM micrograph of the ClpXP1/2 dimer from L. monocytogenes. Some particles are highlighted with ovals. Scale bar, $100 \mathrm{~nm}$ b) Typical reference-free 2D class averages. Arrows indicate additional densities corresponding to ZBDs at the interface between two ClpX hexamers. Scale bar, 20nm c) Ribbon Model of ClpP1 (yellow), ClpP2 (green) and ClpX (orange) superimposed with the cryo-EM density map of the ClpXP1/2 dimer (white and transparent). The upper inset shows the complex shown as slice at the position of the axial pore entry of the upper ClpXP1/2 complex. ClpX and ClpX-ZBD densities are colored magenta and gray transparent, respectively. The arrow indicates the spiral arrangement of the ZBD domains. The lower inset shows four copies of ZBD-dimers (PDB: 1OVX) placed into the cryo-EM density at the interface between the ClpX hexamers. The low resolution density did not allow automated rigid-body fitting, therefore the dimers were placed manually and interconnected as proposed in ${ }^{26}$. d) Cartoon depicting ClpXP1/2 dimerization via the ZBD domains of two opposing ClpX hexamers. Arrows indicate the flexibility of the complex. 

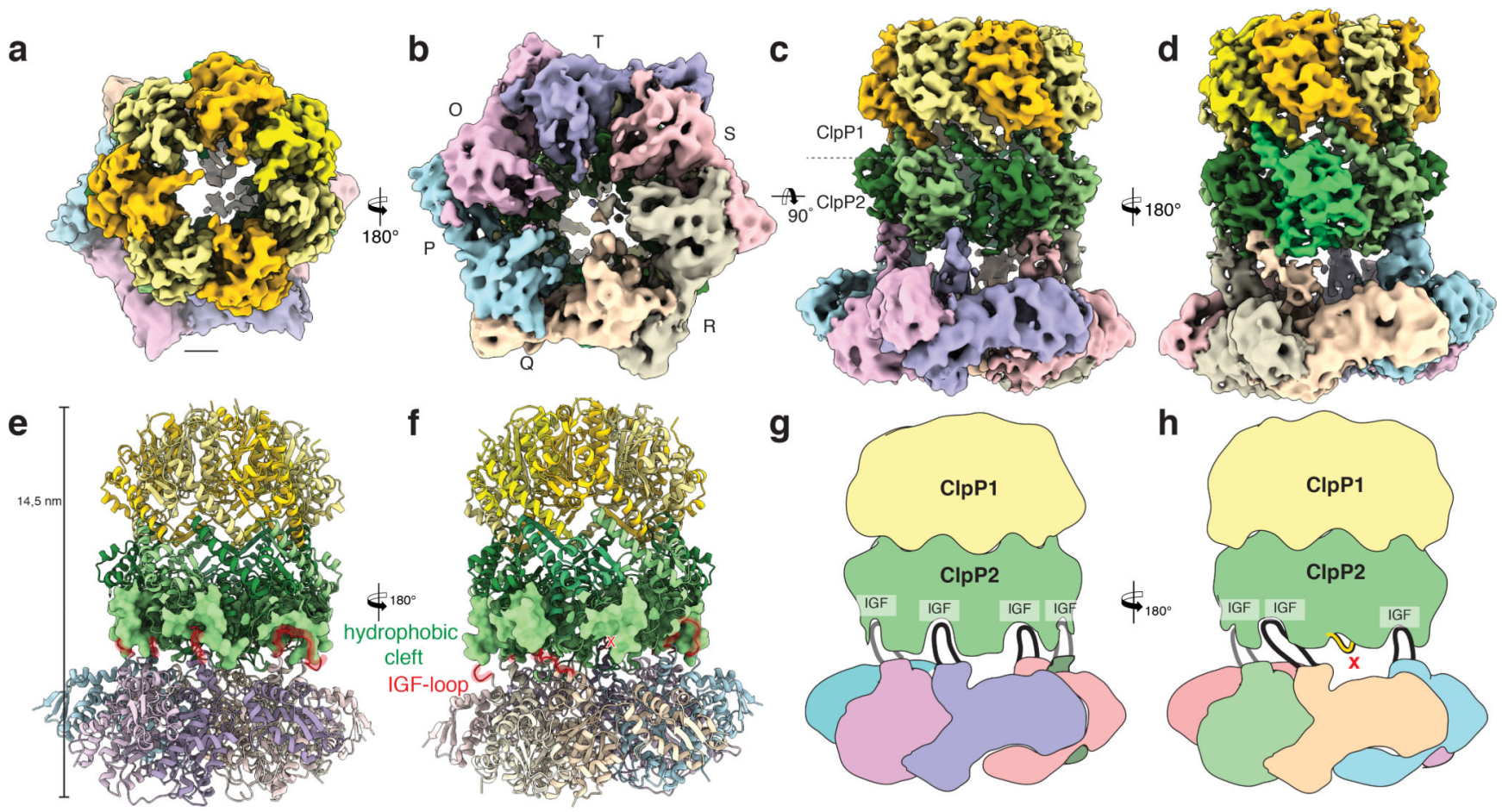

Figure 2. Cryo-EM structure of the ClpXP1/2 protein degradation machinery.

a-d) Cryo-EM density of ClpXP1/2 shown from the top (a), bottom (b) and side (c,d).

$\mathrm{ClpP} 1$ and $\mathrm{ClpP} 2$ subunits are colored in khaki, orange and dark, light green, respectively. $\mathrm{ClpP} 2$ subunit $\mathrm{J}$ is highlighted in mint green. Note that this is the only $\mathrm{ClpP} 2$ subunit not interacting with $\mathrm{ClpX}$ via an IGF-loop. Each subunit of $\mathrm{ClpX}$ is assigned a different color. This color code is maintained throughout the manuscript. e-f) Molecular model of ClpXP. The hydrophobic pockets of ClpP2, each spanning two ClpP2 subunits, are shown as surface. The IGF interaction loops are highlighted in red. g-h) Cartoon depicting how the ClpX hexamer interacts with the ClpP2 heptamer via the six IGF-loops. Note the extended conformation of IGF-loop of ClpX subunit Q. 


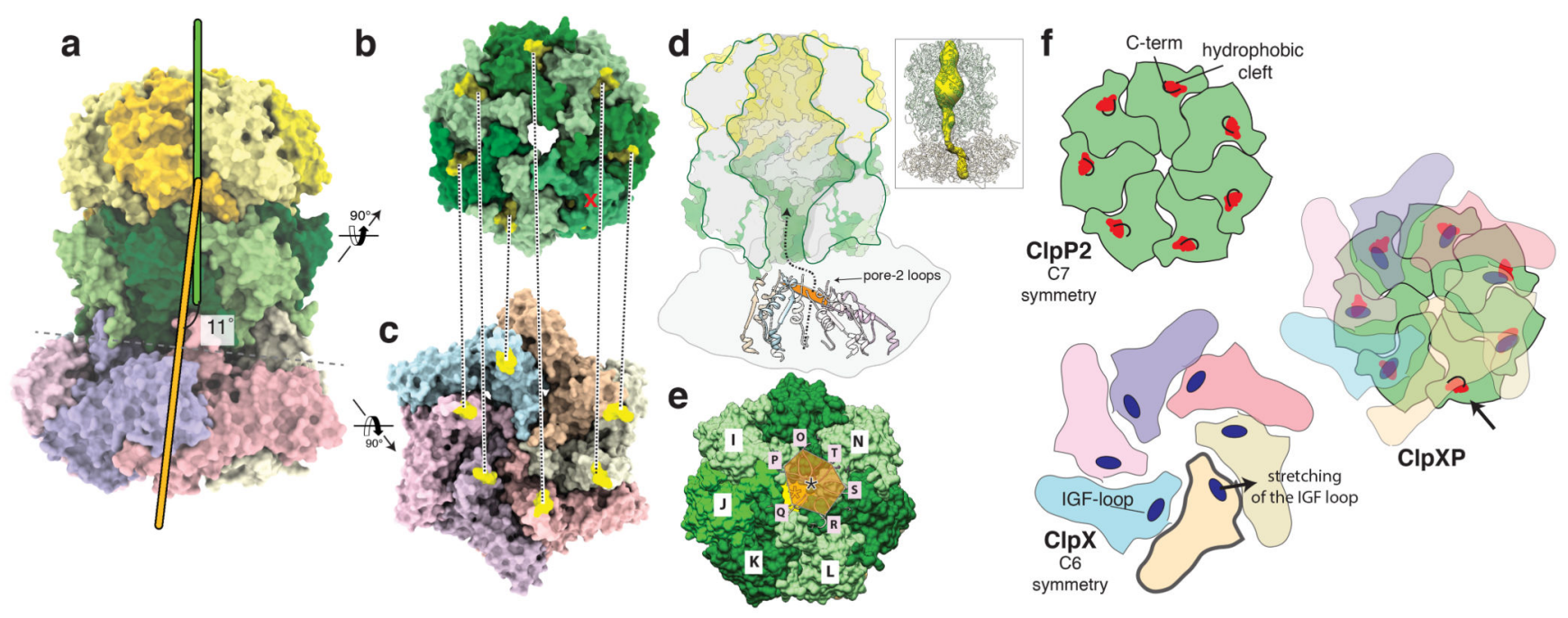

Figure 3. Symmetry mismatch between ClpP1/2 and ClpX.

a) Molecular model of ClpXP1/2. The symmetry axes of the ClpP1/2 and ClpX are shown in green and orange, respectively. b-c) The ClpP2 heptamer (b) and the ClpX hexamer (c) are shown from the bottom and the top, respectively, perpendicular to the plane of the ClpP2ClpX interface. The positions of the IGF-loops and the hydrophobic grooves are highlighted in yellow and connected by dashed lines. d) Cut-away view of the ClpP density. Secondary structure elements directly prior (residues 170-189) and after the pore-2-loops (residues 202-220) of ClpX are shown in ribbon representation. The pore-2-loops are not resolved in the cryo-EM density and not shown here. In order to indicate the arrangement and positioning of the pore-2-loops, as well as the position of the upper opening of the ClpX channel relative to the $\mathrm{ClpP} 2$ pore, a plane was calculated using the $\mathrm{Ca}$ atoms of Gly202 as anchor points and depicted here in orange. The plane is tilted and shifted relative to the ClpP channel axis, suggesting a spiral staircase-like arrangement of the pore-2-loops. The dashed line with the arrowhead indicates the pathway of substrate translocation from ClpX towards the ClpP proteolytic chamber. The inset shows the skin surface of the ClpXP pore. e) Molecular surface of ClpP2 shown from the bottom. Rosetta models of the pore-2-loops of $\mathrm{ClpX}$ are shown as ribbons. The black star indicates the positioning of the ClpX channel opening relative to the ClpP channel opening (yellow star). f) Schematic model of the ClpXClpP2-binding mechanism. Left images depict axial views of the ClpP2 heptamer (green) and the ClpX hexamer prior assembly of the ClpXP protease. The main interaction elements, the ClpX IGF-loops and ClpP2 hydrophobic grooves are highlighted. The remaining "free" ClpP2 hydrophobic groove stays shielded by the respective C-terminus (arrow). 

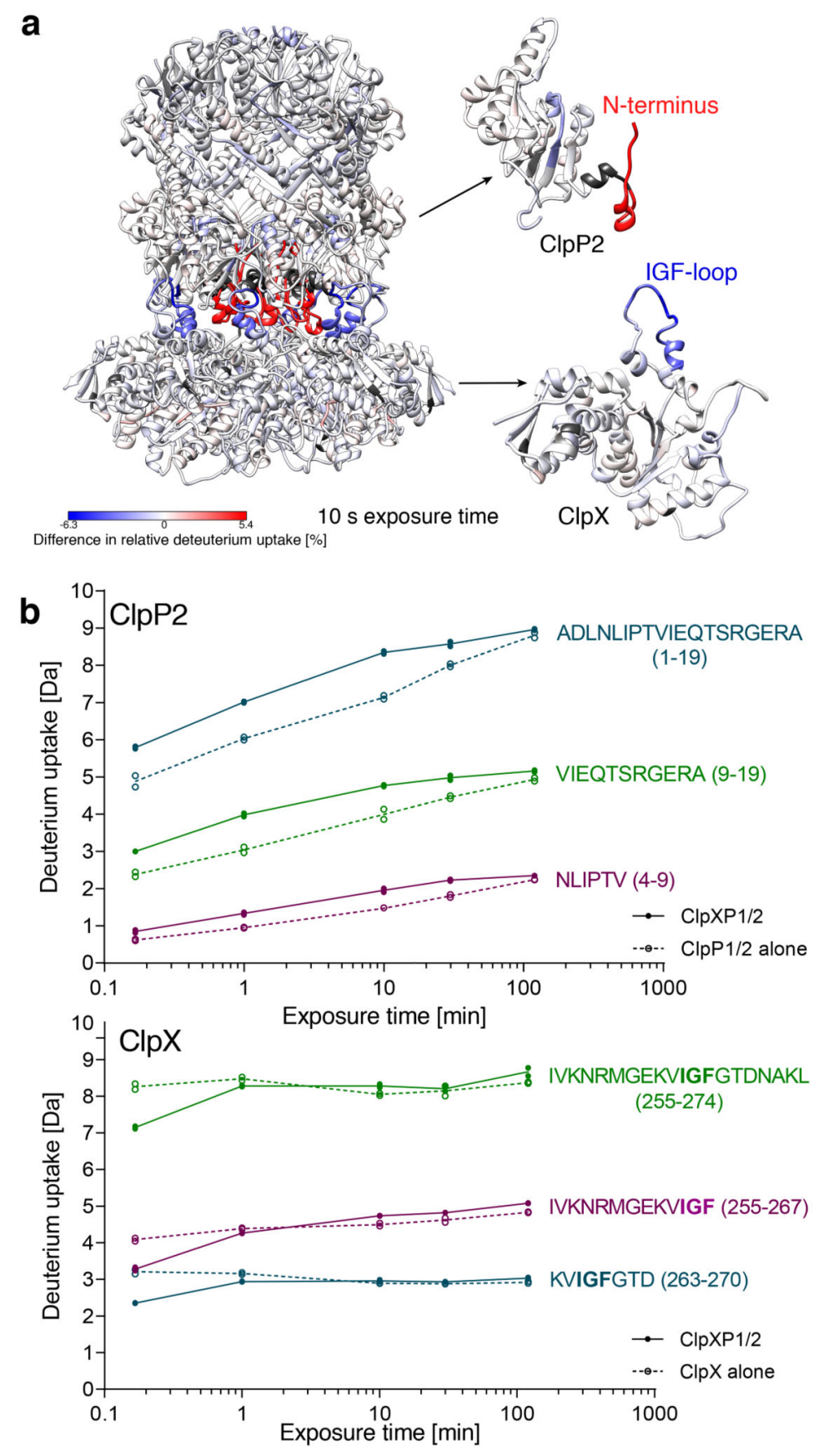

Figure 4. HDX-MS analysis of ClpXP1/2 complex formation.

a) Difference in relative deuterium uptake after $10 \mathrm{~s}$ exposure is mapped on the structure of ClpXP1/2 (left), ClpP2 monomer (right top) and ClpX monomer (right bottom). Increased deuterium uptake upon complex formation is shown in red, decreased deuterium uptake is depicted in blue. Dark gray represents no coverage. The MS data are available online as source data. b) HDX kinetics of exemplary peptides in the N-terminus of ClpP2 (top) and in the IGF-loop of ClpX (bottom). Solid lines and filled circles represent the ClpXP1/2 
complex, dashed lines and empty circles represent $\mathrm{ClpP} 1 / 2$ or $\mathrm{ClpX}$. Two independent replicates are shown, lines denote the mean. 

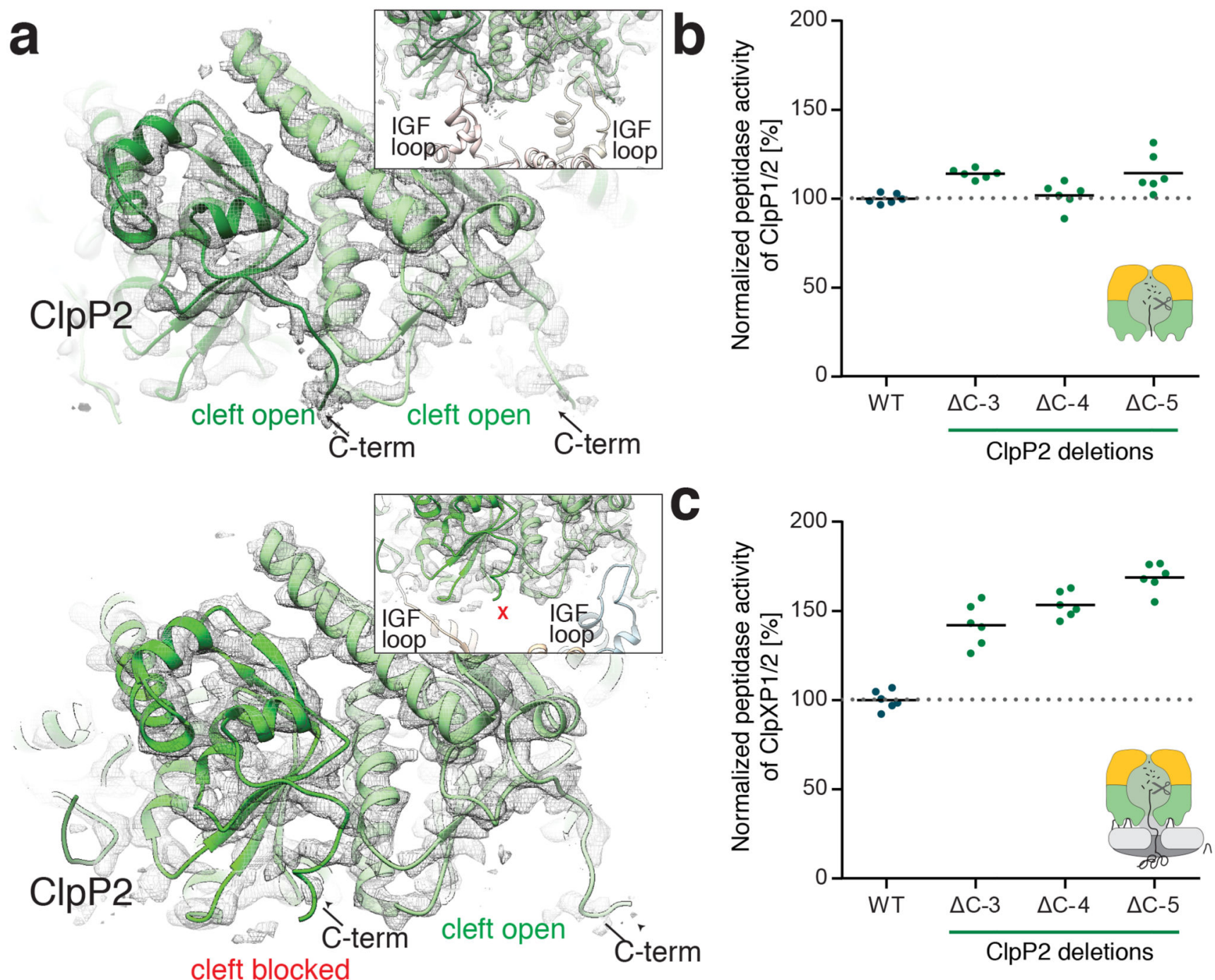

Figure 5. Role of the ClpP2 C-terminus in ClpXP1/2 binding.

a) Molecular model and cryo-EM density of IGF-loop bound (upper image) and not bound to hydrophobic pockets of ClpP2 (lower image). The insets show the respective IGF-loops in ribbon representation. Arrows indicate the C-terminus of ClpP2. b) Peptidase activity of ClpP1/2 with C-terminally truncated ClpP2 (714 nM (ClpP1/2) $14,100 \mu \mathrm{M}$ Ac-Ala-hArg-2Aoc-ACC). c) Protease activity of ClpXP1/2 with C-terminally truncated ClpP2 $(0.2 \mu \mathrm{M}$ $(\mathrm{ClpP} 1 / 2)_{14}, 0.4 \mu \mathrm{M} \mathrm{ClpX}_{6}, 0.8 \mu \mathrm{M}$ GFP-SsrA). Data are normalized to the wild type as $100 \%(n=6$, data were recorded in triplicate and two independent experiments were performed, black lines denote means). Source data for graphs in b-c are available online. 


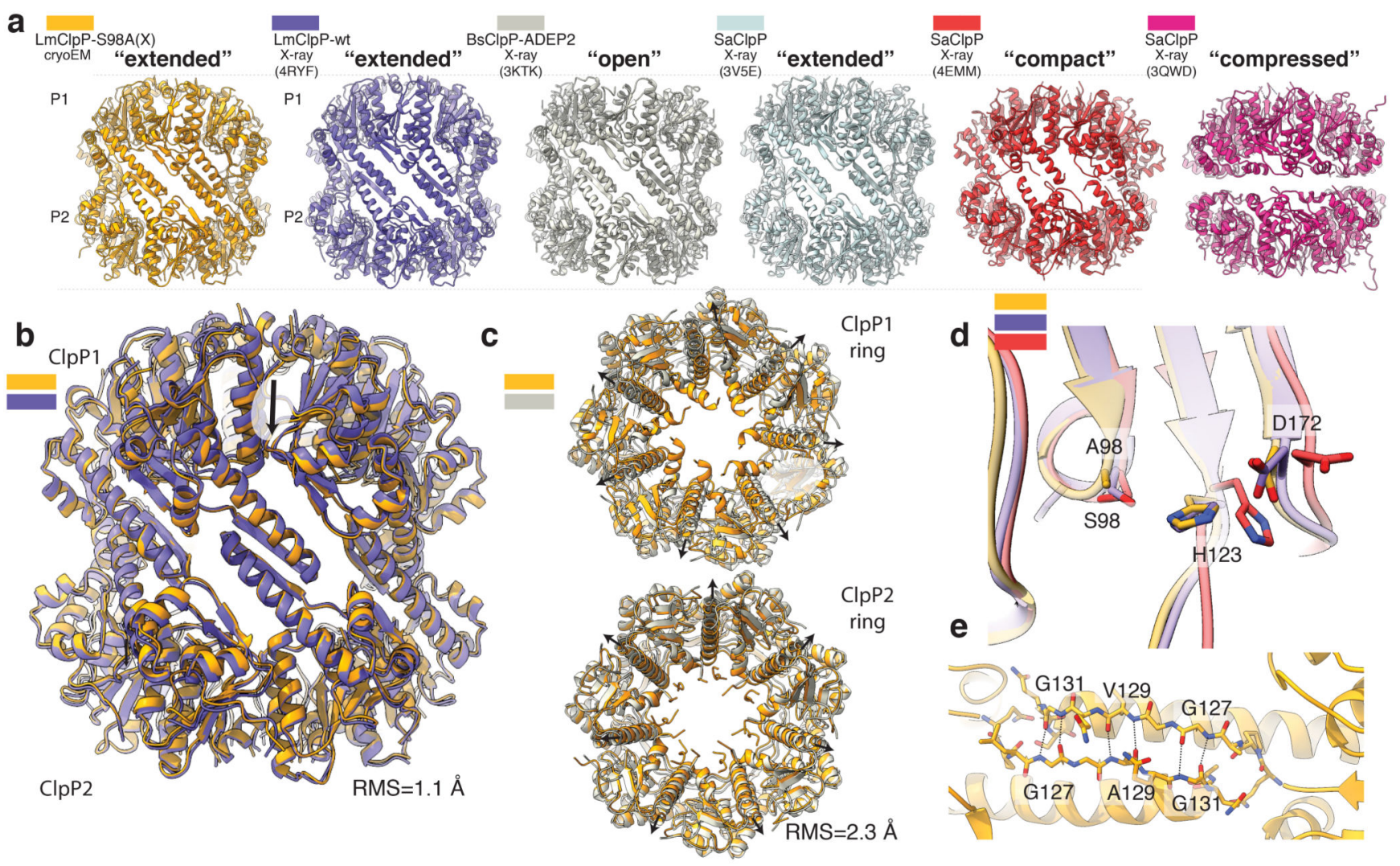

Figure 6. Comparison of ClpX-bound ClpP1/2 with available structures of active and inactive ClpP.

a) Side view of the structure of ClpX-bound LmClpP1/2 (gold) and the crystal structures of LmClpP1/2 in the extended active state (PDB4RYF) (purple), Bacillus subtilis ClpP (BsClpP) in complex with ADEP2 in the extended open active state (PDB 3KTK) (gray), Staphylococcus aureus ClpP (SaClpP) in the extended active state (PDB 3V5E) (cyan), SaClpP in the compact inactive state (PDB 4EMM) (red) and SaClpP in the compressed inactive (PDB 3QWD) (purple) conformation are shown in ribbon representation. b) Structural superposition of ClpX-bound and unbound (PDB 4RYF) LmClpP1/2. The low R.M.S.D suggests that binding of $\mathrm{ClpX}$ to $\mathrm{ClpP} 1 / 2$ does not induce large conformational changes to ClpP1/2. c) Structural superposition of ClpX bound ClpP1/2 heterocomplex and ADEP2-bound ClpP homocomplex (PDB 3KTK) shown in top- and bottom view. Black arrows indicate the characteristic opening of the ClpP pore upon ADEP binding. $\mathbf{d}$ ) Superposition of the catalytic residues S98 (S98A), H123 and D172 (N172) in ClpX-bound LmClpP1-S98A/P2-S98A, LmClpP1/2 (extended active state) (PDB 4RYF), SaClpP (compact inactive state) (PDB 4EMM). Note that despite the S98A mutation, the catalytic residues of ClpX-bound LmClpP1/P2 adopt the active conformation. e) Opposing subunits of ClpX-bound ClpP1 and ClpP2 rings interact via an antiparallel $\beta$-sheet. 


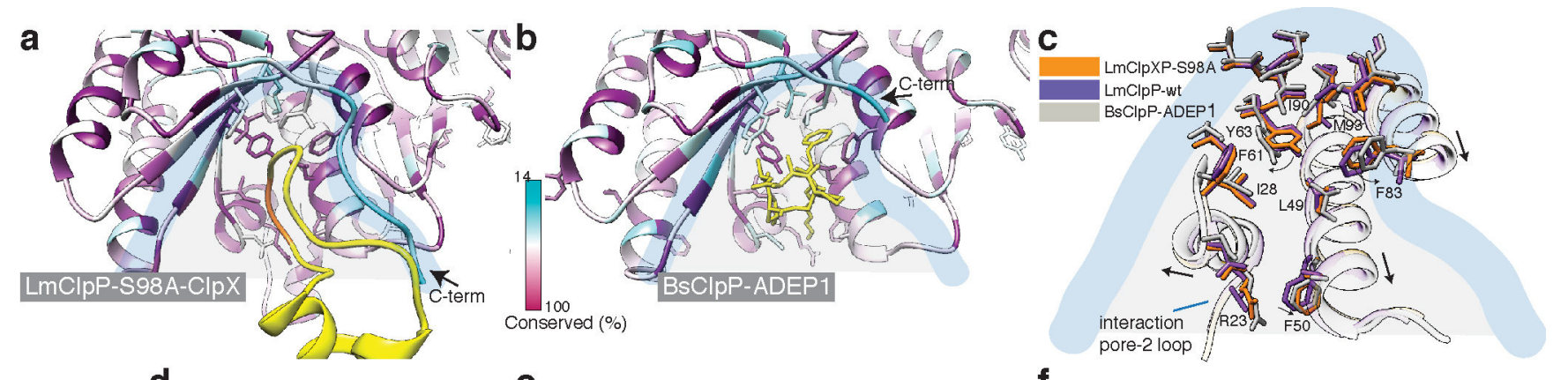

d

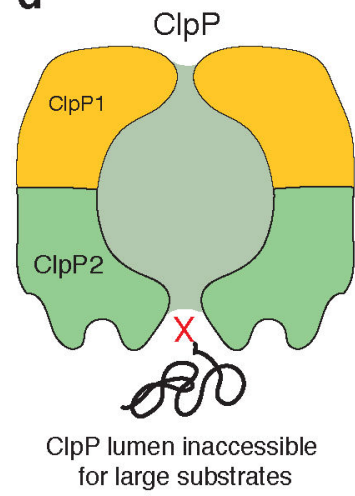

e

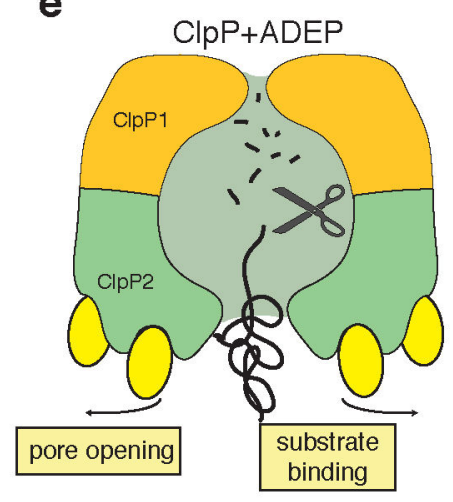

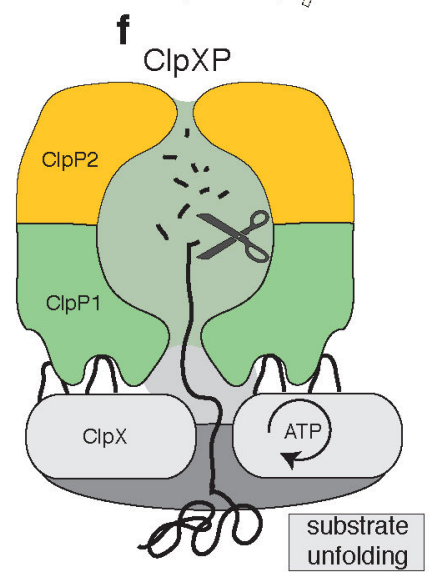

Figure 7. ClpX binds to ClpP in a similar manner like ADEP, but does not induce ClpP pore widening.

a) Local molecular interactions at one of the seven binding pockets between ClpP and the IGF-loop of ClpX. Residues of ClpP are colored by sequence conservation. The IGF-loop is shown in yellow with the IGF residues highlighted in orange. b) Interface of ADEP2 (yellow) with BsClpP (PDB 3KTI) (colored by conservation). c) Structural superposition of the binding pockets of ClpX-bound LmClpP2-S98A, ADEP1-bound BsClpP (PDB 3KTI) and "free" LmClpP2 (PDB 4RYF). Arrows indicate changes upon ADEP binding. d-f) Regulation of ClpP by ClpX and ADEP. The central pore of the ClpP protease is closed and entry of folded proteins into the proteolytic chamber is not allowed (d). ADEP binding to the binding pockets of ClpP induces pore opening. The proteolytic chamber is now accessible for unfolded proteins, leading to unregulated protein degradation and cell death. (e) ClpX binds in the same hydrophobic pockets on ClpP but does not induce pore opening. ClpP and $\mathrm{ClpX}$ form a continuous pore instead, with ClpX unfolding target proteins and forwarding them to the proteolytic chamber of ClpP for degradation in a regulated manner (f). 
Table 1

Cryo-EM data collection, refinement and validation statistics

\begin{tabular}{|c|c|c|c|}
\hline & LmClpX1/2 dimer & \multicolumn{2}{|c|}{$\begin{array}{l}\text { LmClpXP1/2 } \\
\text { EMD-10162, } \\
\text { (PDB-6SFX, 6SFW) }\end{array}$} \\
\hline \multicolumn{4}{|l|}{ Data collection and processing } \\
\hline Magnification & $\mathrm{x} 112,807$ & \multicolumn{2}{|c|}{$\mathrm{X} 112,807$} \\
\hline Voltage $(\mathrm{kV})$ & 300 & \multicolumn{2}{|l|}{300} \\
\hline Electron exposure $\left(\mathrm{e}^{-} / \AA^{2}\right)$ & 114 & \multicolumn{2}{|l|}{114} \\
\hline Defocus range $(\mu \mathrm{m})$ & $0.5--3.0$ & \multicolumn{2}{|c|}{$-0.5--3.0$} \\
\hline Pixel size $(\AA)$ & 1.1 & \multicolumn{2}{|l|}{1.1} \\
\hline Symmetry imposed & $\mathrm{C} 1$ & \multicolumn{2}{|l|}{$\mathrm{C} 1$} \\
\hline Initial particle images (no.) & 273,300 & \multicolumn{2}{|l|}{613,322} \\
\hline Final particle images (no.) & 143,901 & \multicolumn{2}{|l|}{613,322} \\
\hline Map resolution $(\AA)$ & 13 & \multicolumn{2}{|l|}{4} \\
\hline FSC threshold & 0.143 & \multicolumn{2}{|l|}{0.143} \\
\hline Map resolution range $(\AA)$ & - & \multicolumn{2}{|l|}{$3.2-10$} \\
\hline Refinement & & \multicolumn{2}{|c|}{$\underline{6 S F X} \underline{6 S F W}$} \\
\hline Initial model used (PDB code) & - & $4 \mathrm{RYF}$ & - \\
\hline Model resolution $(\AA)$ & - & 2.8 & - \\
\hline FSC threshold & - & - & - \\
\hline Model resolution range $(\AA)$ & - & - & - \\
\hline Map sharpening $B$ factor $\left(\AA^{2}\right)$ & - & -214 & -240 \\
\hline \multicolumn{4}{|l|}{ Model composition } \\
\hline Nonhydrogen atoms & - & 20196 & 15225 \\
\hline Protein residues & - & 2602 & 1955 \\
\hline Ligands & - & - & \\
\hline$B$ factors $\left(\AA^{2}\right)$ & & 100.8 & 187.87 \\
\hline Protein & - & - & \\
\hline Ligand & - & - & \\
\hline \multicolumn{4}{|l|}{ R.m.s. deviations } \\
\hline Bond lengths $(\AA)$ & & 0.012 & 0.018 \\
\hline Bond angles $\left(^{\circ}\right)$ & & 1.233 & 1.978 \\
\hline \multicolumn{4}{|l|}{ Validation } \\
\hline MolProbity score & - & 2.34 & 2.30 \\
\hline Clashscore & - & 22.88 & 22.43 \\
\hline Poor rotamers (\%) & - & 0.18 & 0.2 \\
\hline \multicolumn{4}{|l|}{ Ramachandran plot } \\
\hline Favored (\%) & - & 92.04 & 92.9 \\
\hline Allowed (\%) & - & 7.65 & 6.4 \\
\hline Disallowed (\%) & - & 0.31 & 0.7 \\
\hline
\end{tabular}

